\title{
Intégration financière internationale et croissance économique dans les pays émergents et en développement : le canal du développement financier ${ }^{1}$
}

\author{
Jean-Pierre Allegret ${ }^{\dagger}$ et Sana Azzabi ${ }^{\ddagger}$
}

Résumé : Dans cet article, nous testons les implications de l'ouverture financière sur la croissance de long terme via le canal du niveau de développement financier. Notre travail se démarque de la littérature sur un certain nombre de points, notamment en étudiant le lien entre ouverture financière, développement financier et croissance à l'aide d'un modèle à équations simultanées. A partir de la MMG sur panel dynamique, et pour 112 pays émergents et en développement entre 1975 et 2007, les résultats montrent la présence d'un lien positif entre le développement financier et la croissance de long terme. Contrairement aux résultats d'une abondante littérature étayant un impact positif de l'ouverture financière sur le développement financier, nous aboutissons à une conclusion plus nuancée. Celle-ci est robuste à l'introduction de la variable crises financières. La nature de cet impact dépend du choix de l'indicateur de développement financier. Nos estimations concluent toutefois clairement et dans leur plus grande majorité à des effets négatifs importants de l'ouverture financière sur l'activité des intermédiaires financiers et le développement des marchés boursiers pour les pays émergents et en développement. Les résultats pour le sous-groupe de pays émergents et frontière se révèlent plus concluants. Ils défendent sans ambigüité aucune une relation positive entre l'ouverture financière et le développement financier. Les résultats montrent enfin que le développement financier présente un canal de transmission indirect par lequel l'ouverture financière pourrait impacter le rythme de croissance de long terme.

Mots clés : Intégration financière internationale, Développement financier, Croissance économique, Pays émergents et en développement, Méthode des Moments Généralisés, Panel dynamique, Equations simultanées.

Abstract: In this paper, we test the empirical implications of financial openness on long-term growth through the financial development channel. Our work differs from the existing literature on a number of issues, the most important of them lies in the study of the relationship between financial openness, financial development and growth through the estimation of a simultaneous equations model. Estimates are performed with GMM dynamic panel data techniques for 112 emerging and developing countries from 1975 to 2007. The results confirm the presence of a significant positive relationship between financial development and long term growth. Unlike the results of an extensive empirical literature supporting a significant positive impact of financial openness on financial development, we get a more nuanced conclusion. Such conclusion is robust to the inclusion of the financial crises variable. Indeed, the nature of this impact varies depending on whether we study the implications on the size, on the efficiency or on the activity of financial system. Our estimates suggest however significant adverse effects of financial openness on the financial intermediaries activity and the stock market development in emerging and developing countries. The results for the subgroup of emerging and frontier countries proved more successful and more conclusive. They unambiguously defend a positive and significant link between financial openness and financial development. Finally, the results show that financial development is an indirect transmission channel through which financial openness may impact long-term growth in emerging and developing countries.

Keywords: International financial integration, Financial development, Economic growth, Emerging and developing countries, Generalized Method of Moments, Dynamic panel, Simultaneous equations model.

JEL: C23, E44, F21, F36.

\footnotetext{
${ }^{1}$ Des versions préliminaires de cet article ont été présentées au $2^{\text {ème }}$ colloque international en Finance, Comptabilité et Transparence Financière de l'Association Tunisienne des Sciences de l'Entreprise (ATSE) organisé à Sousse (Tunisie), les 10-11 mai 2013, au 9ème colloque international de l'Association des Economistes Tunisiens organisé à Hammamet (Tunisie), les 06-08 juin 2013, aux 30 èmes journées internationales d'Economie monétaire et financière, colloque annuel du GDRe Monnaie, Banque, Finance, organisées par l’Université de Poitiers à Poitiers (France), les 27-28 juin 2013, et à la $2^{\text {ème }}$ conférence internationale sur les crises de dette et la stabilité financière organisée par l'Université de Toulon à Toulon (France), les 15-16 avril 2014. Nous tenons à remercier les différents participants ainsi que le rapporteur anonyme de la revue pour leurs remarques et suggestions.

† EconomiX - UMR 7235 CNRS et Université Paris Ouest Nanterre La Défense (e-mail: jallegret@u-paris10.fr); Université de Paris Ouest Nanterre La Défense Bâtiment G, bureau 603bis, 200 Avenue de la République, 92001 Nanterre France.

‡ Laboratoire Prospective, Stratégie et Développement Durable (PS2D) - Faculté d’Economie et de Gestion de Tunis, Université de Tunis El Manar, Tunisie. (e-mail: azzabisana@yahoo.fr).
} 


\section{Introduction}

La littérature suggère que les différences de niveau de développement financier entre les pays expliquent celles enregistrées en matière de croissance de long terme. Les travaux théoriques et empiriques ont ainsi souligné les effets positifs du développement financier sur la croissance de long terme (Levine, 2005). Au cours des années quatre-vingt-dix, une extension de cette littérature s'est intéressée aux implications sur la croissance de l'ouverture financière. Cette littérature souligne que les effets positifs des entrées de capitaux ne résident pas uniquement dans leurs effets directs, mais aussi et surtout dans leurs effets induits. Ces avantages «collatéraux potentiels» portent notamment sur le développement du système financier domestique, l'amélioration de la qualité institutionnelle et la discipline des politiques macroéconomiques intérieures (Kose et al., 2009).

Cet article focalise son attention sur l'étude d'un canal particulier de transmission entre l'ouverture financière et la croissance de long terme, à savoir celui du niveau de développement financier. Plus précisément, nous analysons le lien tripartite intégration financière internationale développement financier - croissance de long terme dans les pays émergents et en développement. Notre approche se démarque de la littérature existante en analysant la relation entre les trois variables à partir de l'estimation d'un modèle à équations simultanées. En effet, les investigations existantes tendent à analyser séparément le lien entre l'ouverture financière et le développement financier et celui entre le développement financier et la croissance. L'estimation d'un modèle à équations simultanées permet d'évaluer plus convenablement la nature de la connexion entre l'ouverture financière, le développement financier et la croissance.

Nos investigations prennent en compte les effets potentiels des crises financières sur le rythme de croissance des économies. En effet, une littérature importante suggère que l'absence de prise en compte de l'instabilité macroéconomique qui a accompagné l'ouverture financière dans de nombreux pays peut expliquer la difficulté de détecter un effet causal positif entre l'intégration financière internationale et la croissance. Plus précisément, l'instabilité financière est susceptible de réduire - voire annuler - les effets favorables de l'ouverture financière sur le rythme de la croissance ${ }^{2}$.

Nos principaux résultats sont les suivants. En premier lieu, nous confirmons les conclusions de la littérature quant à la présence d'un lien significativement favorable entre le développement financier et la croissance de long terme, dans les pays émergents et en développement. En deuxième lieu, contrairement aux conclusions d'une abondante littérature étayant des retombées positives significatives de l'ouverture financière sur le développement financier, nos résultats

\footnotetext{
${ }^{2}$ Voir, entre autres, Bussière et Fratzscher (2008), Rancière et al. (2006 et 2008), Guillaumont et Kpodar (2006) et
} Eichengreen et al. (2011). 
nous conduisent à une interprétation plus nuancée. En troisième lieu, l'estimation du modèle à équations simultanées montre que le développement financier présente un canal de transmission indirect par lequel l'ouverture financière pourrait impacter le rythme de croissance de long terme dans les pays émergents et en développement. En quatrième lieu, nos résultats sont robustes à l'intégration de la variable crise financière dans nos estimations.

La suite de l'article est structurée de la manière suivante. La première section présente les fondements théoriques de notre approche. La section deux est consacrée à la présentation de l'échantillon de pays étudiés, au choix des variables et aux méthodes d'estimation utilisées. La troisième section discute du lien empirique développement financier - croissance de long terme. La quatrième section teste l'impact de l'intégration financière internationale sur le développement du système financier domestique. La section cinq estime un modèle à équations simultanées afin d'analyser les implications de l'ouverture financière sur la croissance de long terme via le canal du niveau de développement financier. Une dernière section présente les principales conclusions.

\section{Les interactions entre ouverture financière, développement financier et croissance : une revue de la littérature théorique}

Comme souligné précédemment, cet article s’intéresse à la relation intégration financière internationale, développement financier et croissance de long terme. Cette relation trouve ses fondements théoriques dans la littérature qui s'est d'abord intéressée aux effets du développement financier sur la croissance économique.

Selon Levine (2005 : p.5), le développement financier se définit comme «le processus par lequel les instruments, les marchés et les intermédiaires financiers améliorent le traitement de l'information, la mise en cuvre des contrats et la réalisation des transactions, permettant ainsi au système financier de mieux exercer ses fonctions ». Dans cette perspective, c'est à travers l'exercice plus efficace de leurs fonctions que les systèmes financiers sont en mesure de stimuler l'accumulation du capital et la croissance de la productivité globale des facteurs. Trois aspects sont particulièrement importants pour notre propos. En premier lieu, des systèmes financiers développés permettent de réduire les coûts d'acquisition et de traitement de l'information. Ainsi, dans le cadre d'un modèle de croissance endogène, Greenwood et Jovanovick (1990) considèrent une situation dans laquelle chaque agent peut choisir entre deux types de technologies de production, à savoir une première qui garantit un rendement certain mais relativement faible de l'investissement, et une seconde qui offre un rendement élevé mais plus risqué. En l'absence d'intermédiaires financiers, chaque épargnant doit supporter un coût élevé pour évaluer les entreprises dans lesquelles il désire investir, ou encore les conditions économiques régissant le fonctionnement des marchés. Les épargnants sont ainsi incités à former des coalitions appelées intermédiaires financiers. Ceux-ci prennent en charge le 
processus coûteux d'acquisition et de production de l'information ainsi que celui de coordination des activités productives. Les intermédiaires financiers permettent, ainsi, la réalisation d'économies sur les coûts informationnels. Une importante littérature théorique suggère aussi que les marchés boursiers sont également susceptibles de stimuler la production de l'information. Allen (1993) montre ainsi que lorsque la diversité des opinions est particulièrement élevée entre les investisseurs, les marchés financiers apparaissent mieux à même de les faire converger pour financer les investissements sous-jacents. Allen et Gale (2000) soulignent qu'en situation d'incertitude, les marchés boursiers s'avèrent plus efficaces que les banques dans la collecte et le traitement de l'information. En deuxième lieu, à travers la fonction d'assurance de liquidité, les systèmes financiers permettent d'accroitre le rendement des investissements. Bencivenga et Smith (1991) intègrent dans un modèle de croissance endogène l'idée selon laquelle les investissements réalisés atteignent leur rendement maximum s'ils sont conduits à leur terme. Or, les épargnants peuvent être contraints de liquider leurs placements avant. Il y a ainsi une contradiction entre l'illiquidité des investissements réels et le désir de liquidité des épargnants. En répondant à ces exigences contradictoires, les systèmes financiers engendrent une externalité positive: la minimisation du risque de liquidité par l'intervention des intermédiaires financiers permet de maximiser le volume d'épargne disponible pour l'investissement. En troisième lieu enfin, le système financier joue un rôle important dans la mobilisation et la collecte des épargnes. Acemoglu et Zilibotti (1997) soulignent qu'en mettant en commun l'épargne accumulée par les agents, les intermédiaires financiers financent un plus grand nombre d'investissements. Ils multiplient les possibilités de diversification transversale des risques, ce qui facilite l'allocation des fonds vers les activités les plus rentables et stimule la croissance de la productivité.

L'ouverture financière peut favoriser à la fois la croissance économique et le développement financier. En ce sens, elle peut renforcer les effets de ce dernier sur le rythme de croissance. Une large littérature met en avant le fait que les économies en développement souffrent d'une pénurie de capital et d'une insuffisance d'épargne intérieure (Barro et al. (1995), Rappaport (2000)). Les entrées de capitaux faisant suite à l'ouverture financière vont ainsi favoriser le rythme d'accumulation du capital, et par là-même la croissance. Au-delà de ce canal direct d'accumulation du capital, l'ouverture financière est en mesure de favoriser la croissance de long terme via l'amélioration de l'efficacité allocative des ressources productives et le relèvement du taux de croissance de la productivité globale des facteurs (Gourinchas et Jeanne, 2006). L'ouverture financière exerce un effet positif sur le développement financier à travers l'approfondissement des secteurs bancaires et des marchés boursiers intérieurs (Mishkin, 2009). Plus précisément, la libéralisation du compte financier peut contribuer au développement des systèmes financiers 
domestiques à travers différents canaux (Chinn et Ito (2006), Calderón et Kubota (2009)). Premièrement, l'ouverture financière améliore l'efficacité de l'intermédiation financière et la profondeur des marchés financiers, en éliminant la répression financière. Deuxièmement, la suppression des contrôles sur les mouvements de capitaux internationaux est susceptible d'accroitre les possibilités de diversification de portefeuille tant pour les investisseurs domestiques qu'étrangers, avec des effets positifs sur la taille, la stabilité et la liquidité des marchés boursiers. Troisièmement, une politique d'ouverture financière améliore l'efficience du système financier et ce, par l'élimination d'institutions financières inefficaces et la présence de pressions plus fortes favorables à une réforme de l'infrastructure financière. Ceci est de nature à réduire l'asymétrie d'information (en abaissant la sélection adverse et l'aléa moral) et à accroitre la disponibilité du crédit. Levine (1996) met en avant l'idée selon laquelle l'entrée des banques étrangères est en mesure de promouvoir le développement du secteur bancaire local aussi bien directement, en introduisant des services bancaires de meilleure qualité, qu'indirectement en encourageant le développement des meilleures pratiques de réglementation et supervision. L'entrée des banques étrangères peut également conduire à une amélioration de la qualité et de la disponibilité des services financiers fournis localement, et ce, en stimulant la compétition au sein du secteur bancaire et en réduisant l'influence du gouvernement (Mishkin (2006), Berger et al. (2000)). Aussi, les entrées de capitaux liées à l'ouverture financière sont susceptibles d'améliorer la liquidité des marchés financiers et réduire, par la même, la prime de risque (Levine et Zervos, 1998a). L'efficience des marchés peut être améliorée à travers un accroissement de la quantité et de la qualité de l'information disponible sous l'influence de la présence d'investisseurs étrangers (Jain-Chandra, 2002). En outre, la participation d'investisseurs étrangers est de nature à accroître la liquidité au sein de marchés jusque-là faiblement liquides, avec des effets positifs indirects sur l'efficacité du marché (Demirguç-Kunt et Levine (1996), Kim et Singhal (2000)).

\section{Estimation économétrique}

Après avoir présenté de manière successive l'échantillon, la période d'analyse et les variables utilisées, la méthodologie économétrique est introduite.

\subsection{Echantillon et période d'analyse}

Nous mettons en œuvre des estimations économétriques portant sur un panel de 112 pays émergents et en développement entre 1975 et 2007 (voir liste A de l'annexe 2 pour la composition détaillée de l'échantillon de pays). Nous utilisons la classification de la Banque Mondiale de juillet 2008 qui distingue les pays en fonction du Revenu National Brut (RNB) par habitant de l'année 2007, et retenons : les pays à faible revenu (RNB par habitant inférieur ou égal à 935\$), les pays à revenu moyen inférieur (RNB par habitant compris entre $936 \$$ et $3705 \$$ ), et 
les pays à revenu moyen supérieur (RNB par habitant compris entre $3706 \$$ et 11 455\$). La période d'étude est dictée par la disponibilité des données. Les données utilisées sont calculées en moyenne sur sept sous-périodes non chevauchées de cinq années chacune ${ }^{3}$.

\subsection{Présentation des variables}

\subsubsection{Le développement financier (DF)}

Quatre catégories d'indicateurs financiers sont retenues ici. La première concerne des indicateurs de taille (absolue et relative) des intermédiaires financiers : le ratio des passifs liquides du système financier rapportés au PIB (lly), le ratio des actifs des banques commerciales sur la somme de ces actifs plus ceux de la banque centrale $(d b c b a)$, et le ratio de l'actif total des banques de dépôts rapporté au PIB (dbay). La deuxième catégorie porte sur des mesures du niveau d'activité des intermédiaires financiers : le crédit au secteur privé accordé par les banques de dépôts sur le PIB (privdby), le crédit au secteur privé accordé par les banques commerciales et autres institutions financières sur le PIB (privy), les dépôts des banques commerciales rapportés au PIB (bdy), et les dépôts du système financier sur le PIB $(f d y)$. La troisième catégorie s'intéresse à des indicateurs d'efficacité du secteur bancaire : le ratio des frais généraux des banques (overhead) et celui de la marge nette d'intérêts (margin). Enfin, un certain nombre d'indicateurs de développement du marché boursier sont retenus : le ratio de capitalisation boursière (cap), le ratio de la valeur des actions échangées (traded), le taux de rotation des actifs (turnover) et le nombre de firmes cotées par habitant (list).

Les données relatives à ces indicateurs proviennent de la base de données élaborée par Beck, Demirgüç-Kunt et Levine en 2000 et mise à jour en novembre $2010^{4}$.

\subsubsection{La variable de l'intégration financière internationale $(\mathrm{OF})$}

Nous employons deux catégories de mesures du degré d'intégration financière internationale: une mesure de jure - l'indice de Chinn et Ito de libéralisation du compte financier (kaopen) - et des mesures de facto - la somme rapportée au PIB des stocks bruts d'avoirs et d'engagements extérieurs (assetliab), la valeur rapportée au PIB des stocks bruts d'avoirs extérieurs (asset) et la

\footnotetext{
${ }^{3}$ Les sous-périodes distinguées dans l'analyse sont les suivantes: 1975...1979, 1980..1984, 1985...1989, 1990...1994, 1995_..1999, 2000...2004, 2005...2007. Seule la dernière sous-période est composée de seulement 3 ans au lieu de 5 ans.

${ }^{4}$ Pour plus de détails sur la définition de ces indicateurs, voir Beck et Demirgüç-Kunt (2010). Les données concernant d'autres indicateurs de développement du système financier, ceux se rapportant - à titre d'exemple - au développement des marchés obligataires (ratio de capitalisation du marché obligataire public, celui de capitalisation du marché obligataire privé...) ou à l'activité d'autres institutions financières (les compagnies d'assurance notamment), ne sont pas disponibles pour beaucoup de pays de l'échantillon et sur une longue période de temps, ce qui empêche leur utilisation dans ce genre d'estimations. Avec ces indicateurs, le nombre d'observations devient tellement faible qu'il rend impossible l'obtention de résultats intéressants pour ces dimensions du développement financier.
} 
valeur rapportée au PIB des stocks bruts d'engagements extérieurs (liab) ${ }^{5}$. Les données relatives aux stocks bruts d'avoirs et d'engagements extérieurs sont issues de la base de Lane et MilesiFerretti (2007)

\subsubsection{Les variables de contrôle}

En nous appuyant sur la littérature, nous tenons compte d'un ensemble de variables de contrôle. Ces dernières diffèrent selon les estimations économétriques. Ainsi, dans la première investigation consacrée au lien développement financier - croissance économique, nous retenons : le PIB réel initial par habitant (PIB initial) qui reflète la prise en compte de la convergence conditionnelle, le degré de développement éducatif (prim) pour mesurer le stock de capital humain, le taux d'inflation domestique (inf) et le taux des dépenses publiques en pourcentage du PIB (gov) comme indicateurs de la stabilité macroéconomique (Easterly et Rebelo (1993), Fischer (1993)), le degré d'ouverture commerciale (trade) comme indicateur global des distorsions au commerce, la prime du marché de change parallèle $(b m p)$ comme indicateur global des distorsions intérieures dans l'économie (Dollar, 1992) et, enfin, le degré de développement des institutions locales (bq, cor, invt, libciv, libpol) comme préconisé par une vaste littérature économique (La Porta et al. (1997, 1998), Acemoglu et al. (2005)).

Pour la deuxième investigation empirique consacrée au lien ouverture financière - développement financier, les variables de contrôle sont les suivantes : le PIB réel par habitant (ycap) et le taux d'inflation (inf) afin de rendre compte des effets de l'environnement macroéconomique sur le développement financier (Huybens et Smith (1999), Chinn et Ito (2002, 2006), Khan et al. (2006)), et le degré d'ouverture commerciale (trade) et le taux de développement institutionnel (risque politique, creditor, instit) comme des déterminants potentiels du développement financier par référence aux travaux de Rajan et Zingales (2003), Svaleryd et Vlachos (2002)....

Une description détaillée des variables utilisées ainsi que les sources sont présentées dans le tableau 1 de l'annexe $1^{7}$.

\footnotetext{
5 Pour avoir plus de détails sur l'indice kaopen ainsi que sur la base de données utilisée, voir le lien http://web.pdx.edu/ ito/Chinn-Ito website.htm.

${ }^{6}$ Selon Baltagi et al. (2009), les mesures de facto sont moins concernées par le problème d'endogénéité que les mesures de jure. D'après ces auteurs, la décision officielle (de jure) d'ouverture ou de fermeture de l'économie nationale aux flux financiers transfrontaliers peut être fortement influencée par le comportement des groupes d'intérêt. Au contraire, bien qu'elles soient influencées par les politiques nationales, les mesures de facto reflètent aussi des facteurs tels que l'histoire du pays, sa géographie, les politiques internationales.... Ces déterminants fixes (exogènes) sont, bien entendu, hors du contrôle des décideurs nationaux, et donc moins affectés par les politiques des groupes d'intérêt. Bien que cela puisse rendre les mesures de facto plus appropriées pour une étude de la relation entre l'ouverture financière et le développement financier, Baltagi et al. (2009) reconnaissent que toute discussion concernant les avantages et les inconvénients des mesures de l'ouverture financière est difficile à clôturer en l'absence d'une réelle modélisation théorique.

7 Tous les tableaux figurent en annexe.
} 


\subsection{Méthodologie économétrique}

Les deux premières estimations empiriques - relations ouverture financière - développement financier et développement financier - croissance économique de long terme - sont effectuées avec la Méthode des Moments Généralisés (MMG) sur panel dynamique. Cette méthode permet en effet de traiter le problème d'endogénéité potentielle de l'ensemble des variables explicatives des modèles estimés (non seulement celui de la variable d'intérêt comme c'est le cas avec la technique des variables instrumentales). Elle présente, par ailleurs, l'avantage de générer des instruments internes à partir des variables explicatives endogènes du modèle. L'estimateur utilisé est celui de la MMG en système proposé par Blundell et Bond (1998). Cet estimateur combine, dans un seul système, le modèle en niveau avec celui en différence première ${ }^{8}$. Les instruments pour la régression en différence sont ceux préconisés par Arellano et Bond (1991). On instrumentalise ainsi les différences premières des variables explicatives endogènes du modèle par leurs valeurs retardées (d'au moins deux périodes) en niveau. Les variables explicatives endogènes du modèle en niveau sont, quant à elles, instrumentées par leur différence première la plus récente, et ce, sous l'hypothèse de « quasi stationnarité » de ces variables'. Le système d'équations ainsi obtenu est estimé à l'aide de la MMG. Les estimations effectuées correspondent à la procédure d'estimation en deux étapes. Lors de la première étape, la structure d'auto-corrélation des perturbations est prise en compte et les termes d'erreur sont supposés être indépendants et homoscédastiques aussi bien dans le temps qu'entre les individus. Le vecteur des résidus estimés est utilisé, au cours de la seconde étape, afin d'estimer de façon convergente la matrice de variances-covariances des perturbations. Nous avons préféré cette procédure d'estimation à l'estimation en une étape étant donné sa plus grande efficacité asymptotique (Sevestre (2002), Roodman $(2006,2009))^{10}$. Afin de tester la validité des instruments utilisés, nous avons eu recours au test de Hansen (1982) de sur-identification des restrictions sur les moments, tel que suggéré par Arellano et Bond (1991), Arellano et Bover (1995) et Blundell et Bond (1998). Il permet de tester la validité des valeurs retardées - en niveau et en différence - comme instruments (Kpodar, 2007). Nous avons également procédé au test d'auto-corrélation des erreurs d'Arellano et Bond (1991). Celui-ci teste la corrélation sérielle de premier ordre des résidus en niveau en estimant la corrélation sérielle de second ordre des erreurs en différence.

\footnotetext{
8 A partir d'une étude de simulation, Blundell et Bond (1998) montrent que l'inclusion de la régression en niveau améliore sensiblement la qualité de l'estimation et réduit les biais potentiels associés à l'estimateur en différence.

${ }^{9}$ Selon cette hypothèse, la corrélation entre l'effet individuel et les variables explicatives endogènes du modèle en niveau est constante dans le temps.

${ }^{10}$ Par ailleurs, afin de limiter le nombre d'instruments utilisés et éviter les biais de sur-instrumentation du modèle, nous avons employé la commande « collapse » du logiciel utilisé pour l'estimation (Roodman, 2006 et 2009). Roodman (2009) démontre la supériorité de la commande « collapse » à partir d'études de simulations.
} 
L'estimateur MMG en système de Blundell et Bond (1998) que nous employons dans cette étude présente un certain nombre de limites. Cet estimateur est non approprié pour des panels non stationnaires. C'est précisément ce problème que les techniques économétriques comme la méthode de Pesaran et Smith (1995) (estimateur de moyenne groupée, MG), ou celle de Pedroni (1996, 2000) (technique des moindres carrés ordinaires modifiés, Fully Modified Ordinary Least Squares, FMOLS) tentent de pallier. Ces techniques sont bien adaptées pour les données macroéconomiques qui sont non stationnaires. Elles ne sont, toutefois, pertinentes que dans le cas de panels cylindrés. Ceci n'est pas le cas du notre, ce qui justifie la non utilisation de ces méthodes économétriques - même si elles sont plus adaptées - lors de nos estimations empiriques.

Les estimations du modèle à équations simultanées décrivant la relation tripartite ouverture financière - développement financier - croissance sont effectuées moyennant la technique des triples moindres carrés. Plus précisément, une grande variété de méthodes a été proposée pour estimer les modèles à équations simultanées. La méthode des Moindres Carrés Ordinaires (MCO), généralement utilisée, ne permet pas de donner un estimateur consistant et sans biais dans la mesure où elle ne prend pas en compte le problème d'endogeneité potentielle des variables. Il y a cependant des cas où cette méthode conduit à des estimations correctes, notamment lorsque les modèles sont récursifs. La méthode des Double Moindres Carrés (DMC) prend en considération le problème d'endogeneité et offre, sous des hypothèses assez générales, un estimateur DMC consistant. Cette propriété disparaît, en revanche, dans le cas, fréquent, d'endogènes retardées et d'autocorrélation de l'aléa ${ }^{11}$. Les méthodes précédentes sont des techniques d'estimation dites équation par équation, et ce, contrairement à la méthode des triples moindres carrés - mathématiquement plus complexe - qui estime globalement l'ensemble des équations d'un modèle. La technique des triples moindres carrés commence par estimer chaque équation par les DMC (ou les variables instrumentales), puis utilise les résidus de cette première étape pour estimer la liaison entre les aléas des différentes équations et utilise enfin les moindres carrés généralisés (MCG) pour estimer globalement l'ensemble du modèle en tenant compte de cette information. L'avantage de cette technique, comparativement aux autres, réside ainsi dans la prise en compte des corrélations entre les termes d'erreur, ainsi que des problèmes d'endogeneité et de simultanéité. Elle est, par ailleurs, la plus adaptée au cas de panels non-cylindrés que nous sommes contraints d'utiliser vu l'indisponibilité des données.

\footnotetext{
${ }^{11}$ L'application de cette technique à des données de panel est par ailleurs assez délicate étant donné l'existence d'une double dimension : temporelle et individuelle.
} 


\section{Relation développement financier et croissance économique de long terme}

\subsection{Modèle testé}

Afin d'examiner la relation entre le développement financier et la croissance économique de long terme, nous estimons le modèle spécifié dans l'équation suivante :

$$
y_{i t}-y_{i t-1}=\alpha_{0}+\gamma y_{i t-1}+\alpha_{1} \boldsymbol{D} \boldsymbol{F}_{i t}+\alpha_{2} X_{i t}+\mu_{i}+\lambda_{t}+\varepsilon_{i t}
$$

avec $y$, le PIB réel par habitant exprimé en logarithme, DF, le degré de développement financier, $X$ un jeu de variables de contrôle spécifiées précédemment ${ }^{12}, \mu_{i}$ l'effet spécifique au pays, $\lambda_{t}$ l'effet spécifique au temps et $\varepsilon$ le terme d'erreur. Les indices $i$ et $t$ désignent respectivement les pays $(i=1,2 \ldots N)$ et les périodes $(t=1,2 \ldots T)$.

\subsection{Principaux résultats}

Les tableaux 2 et 3 fournissent les résultats des estimations de l'équation (1). Ils confirment les prévisions de la littérature étudiant le lien entre le développement financier et la croissance de long terme (King et Levine (1993 a et b), Levine (1999), Beck et al. (2000), Benhabib et Spiegel (2000), Levine et al. (2000)) $)^{13}$. Le développement de l'intermédiation financière présente ainsi un coefficient significativement positif au seuil d'erreur de $1 \%$ et ce, quelle que soit la mesure de développement financier utilisée. Ce coefficient suggère qu'une augmentation de $10 \%$ du niveau de développement des intermédiaires financiers provoque une hausse de la croissance du PIB réel par habitant comprise entre 1,08\% (hausse la plus faible obtenue suite à l'accroissement du taux de crédit bancaire au secteur privé) et 2,1 \% (hausse la plus élevée procurée par le relèvement de l'importance des banques commerciales comparativement à la banque centrale dans l'allocation de l'épargne). Une meilleure efficience des banques domestiques (i.e. de faibles ratios de frais généraux et/ou marge nette d'intérêts) semble également favoriser significativement la croissance (coefficients négatifs pour ces deux indicateurs d'efficacité bancaire). Le développement du marché boursier importe aussi significativement dans le processus de croissance des pays émergents et en développement et ce, conformément aux résultats de la littérature (Levine et Zervos (1998b), Rousseau et Wachtel (2000), Beck et Levine (2004), Saci et al. (2009)). La taille (capitalisation boursière) et l'activité (valeur des actions échangées) de ce marché présentent toutes les deux une corrélation significativement positive (au seuil de $5 \%$ ) avec la croissance du PIB réel par habitant. Le degré de rotation des actifs (mesure du niveau d'efficacité du marché boursier) ne semble pas, en revanche, impacter significativement sur le rythme de croissance au

\footnotetext{
${ }^{12}$ L'ensemble de ces variables est exprimé en logarithme, ce qui permet d'interpréter les coefficients estimés du modèle comme des élasticités.

${ }^{13}$ Le test de Hansen (1982) de sur-identification des restrictions sur les moments et le test d'auto-corrélation des erreurs d'Arellano et Bond (1991) montrent que nous ne pouvons rejeter l'hypothèse nulle de validité des instruments utilisés ainsi que celle d'absence de corrélation sérielle d'ordre 2 des termes d'erreur.
} 
sein de ces économies ${ }^{14}$. Le lien entre le développement du système financier et la croissance de long terme semble ainsi être important.

Vu l'importance des institutions pour la promotion de la croissance, nous reportons - au niveau des tableaux 4 et 4bis - les résultats des estimations après contrôle d'un jeu riche et varié de mesures de développement institutionnel. Il s'agit notamment: de la qualité de la bureaucratie (bq), du niveau de corruption du système politique (cor), du profil d'investissement (invt), de l'indice des libertés civiles (libciv) et de l'indice des droits politiques (libpol). Une large littérature économique défend l'importance de la contribution du développement institutionnel dans le processus de croissance (Shleifer et Vishny (1993), Mauro (1995), La Porta et al. (1997, 1998), Rodrik (2003), Acemoglu et al. (2005)). Les résultats des estimations montrent un coefficient significativement positif de la variable « développement financier» dans tous les cas étudiés. Ces résultats viennent conforter davantage la primauté du rôle que peut jouer le système financier dans l'accélération du rythme de croissance au sein des pays émergents et en développement ${ }^{15}$.

En ce qui concerne les variables de contrôle, le coefficient significativement négatif (au seuil d'erreur de $1 \%$ ) que présente la valeur initiale du PIB réel par habitant est conforme à la théorie de convergence conditionnelle. Lorsque le développement du système financier est saisi par celui de l'intermédiation financière, nous remarquons que le niveau d'éducation, le taux de dépenses gouvernementales et la prime du marché des changes parallèle entrent dans la régression de croissance avec des coefficients bien souvent significatifs et conformes à la théorie. En revanche, l'inflation et l'ouverture commerciale ne semblent pas affecter, d'une manière substantielle, le taux de croissance économique de long terme, puisque, dans presque aucun des cas étudiés, elles ne présentent de coefficients significatifs. Les coefficients des variables de contrôle sont beaucoup moins significatifs lorsque le niveau de développement financier est saisi par celui du marché boursier local (tableau 3). Pour ce qui est des variables institutionnelles (tableaux 4 et 4bis), seule la variable saisissant le risque pesant sur l'investissement (" profil d'investissement ») semble impacter d'une manière statistiquement significative sur la croissance du PIB réel par habitant. En revanche et contrairement à ce qui est attendu, aucune des autres mesures de la qualité institutionnelle n’a montré de coefficients significatifs. Le développement du système financier semble ainsi, d'après l'ensemble de ces résultats, importer plus dans le processus de croissance des pays émergents et en développement que la qualité des institutions.

\footnotetext{
14 D'après les résultats des estimations, on remarque que l'impact sur la croissance du développement de l'intermédiation financière est plus large et statistiquement plus significatif que celui du développement du marché boursier. En termes chiffrés, les estimations révèlent qu'une hausse de $10 \%$ du degré de développement de l'intermédiation financière relèverait de plus de $1 \%$ le taux de croissance du PIB réel par habitant, contre seulement près de $0,5 \%$ (presque la moitié) pour le développement du marché boursier.

15 Toutes les spécifications figurant au niveau des tableaux 4 et 4 bis sont exemptes du risque d'auto-corrélation sérielle d'ordre (2) des termes d'erreur ainsi que de celui de non validité des instruments utilisés.
} 


\subsection{Analyse de robustesse}

Nous testons la robustesse des résultats en introduisant de nouvelles variables de contrôle, en éliminant les observations aberrantes du développement financier et en modifiant l'échantillon de pays considérés ${ }^{16}$.

Les estimations sur panel dynamique de l'équation (1) ont été refaites en introduisant de nouvelles variables de contrôle. Celles-ci sont susceptibles d'altérer le pouvoir explicatif du développement financier dans le modèle estimé. Il s'agit de la croissance de la population ( $\not \circ p)$ et de la variation des termes de l'échange (te) (Levine et al., 2000). Les résultats des estimations montrent que l'introduction de nouvelles variables de contrôle n'affecte nullement nos précédentes conclusions. Le coefficient de la variable développement financier demeure significativement positif.

Afin de nous assurer que cet impact positif ne relève pas de l'effet des observations aberrantes, nous restreignons l'échantillon aux observations du développement financier pour lesquelles l'écart (en valeur absolue) du taux de croissance de celui-ci $(d(D F))$ par rapport à sa moyenne $\left(\mu_{d(D F)}\right)$ ne dépasse pas deux fois son écart type $\left(\sigma_{d(D F)}\right)$ et ce, comme suit : $\mid d(D F)-$ $\mu_{d(D F)} \mid<2 . \sigma_{d(D F)}$. Les résultats confirment la robustesse des conclusions précédentes. Le coefficient estimé de la variable développement financier apparait, pour l'ensemble des cas étudiés (i.e. quel que soit l'indicateur de développement financier utilisé), significativement positif (au seuil de $1 \%$ ) et il est compris dans la fourchette attendue.

Afin de confirmer que nos résultats ne proviennent pas de la nature de l'échantillon étudié, nous procédons à la réestimation de l'équation (1) pour un échantillon composé de 30 pays émergents et frontière (voir liste B de l'annexe 2) selon la classification proposée par MSCI Barra. Les pays émergents et frontière se caractérisent par des systèmes financiers développés, et surtout par des marchés boursiers relativement actifs et ce, comparativement aux autres pays en développement pris en compte dans l'échantillon étudié. A nouveau, les estimations du modèle de base se montrent robustes. Les résultats mettent en avant l'effet significativement positif du développement du système financier - dans ses deux composantes intermédiaires financiers et marché boursier - sur la croissance de long terme des pays émergents et frontière. La taille (capitalisation boursière) et la liquidité (valeur des actions échangées) de la bourse jouent, par ailleurs, un rôle positif statistiquement plus significatif - dans le processus de croissance de ces économies - comparativement à la taille et à la liquidité des intermédiaires financiers. Ce résultat témoigne du rôle de plus en plus important des marchés boursiers dans le financement et le développement de ces pays.

\footnotetext{
${ }^{16}$ Les résultats ne sont pas reproduits mais sont disponibles auprès des auteurs sur simple demande.
} 
$\mathrm{Au}$ terme de l'ensemble de ces analyses de robustesse, nous pouvons conclure que le développement financier semble constituer un moteur important de la croissance économique de long terme dans les pays émergents et en développement.

\section{Intégration financière internationale et développement du système financier domestique}

Nous étudions les implications empiriques d'une variation du degré d'intégration financière internationale sur celui du développement du système financier domestique dans sa conception la plus large (intermédiation financière et marché boursier). La littérature empirique privilégie pour l'essentiel des approches microéconomiques afin d'étudier la question des liens entre ouverture financière et développement financier. Nous adoptons ici une approche macroéconomique.

\subsection{Modèle estimé}

En nous inspirant de la littérature (Chinn et Ito (2002), Baltagi et al. (2009), Calderón et Kubota (2009)), nous estimons le modèle suivant :

$$
D F_{i t}=\beta_{0}+\tau D F_{i t-1}+\beta_{1} \boldsymbol{O} \boldsymbol{F}_{i t-1}+\beta_{2} Z_{i t-1}+\mu_{i}+\lambda_{t}+\zeta_{i t}
$$

$D F$ représente le degré de développement financier, $O F$ est une mesure du degré d'ouverture financière et $Z$ est un jeu de variables de contrôle ${ }^{17}$. $\mu_{i}$ représente l'effet spécifique au pays, $\lambda_{t}$ l'effet spécifique au temps et $\zeta$ est un terme d'erreur. Les indices $i$ et $t$ désignent respectivement les pays $(i=1,2 \ldots \mathrm{N})$ et les périodes $(\mathrm{t}=1,2 \ldots \mathrm{T})$. De la même façon que Chinn et Ito (2002, 2006), Law et Demetriades (2004), Huang (2006) et Baltagi et al. (2009), nous tenons compte de l'histoire passée du développement financier en tant que déterminant du développement financier actuel. Nous considérons, par ailleurs, les valeurs retardées des différentes variables explicatives et ce, afin d'éviter tout biais dû à un problème de causalité inverse ou de simultanéité. Comme précisé auparavant, la technique d'estimation employée est celle des moments généralisés sur panel dynamique en système de Blundell et Bond (1998).

\subsection{Principaux résultats}

Les tableaux 5 et 6 présentent les résultats des estimations sur panel dynamique de l'équation $(2)^{18}$. Le tableau 6 expose les résultats des estimations après prise en compte de trois variables ayant trait au développement de l'environnement institutionnel. Il s'agit de l'indice de risque politique (risque politique), de l'indice de protection des droits des créanciers (creditor) et d'un indice composite (instit) prenant en compte la corruption, la démocratie, la qualité de la bureaucratie, le respect des lois et la stabilité du gouvernement (voir tableau 1). Le rôle des institutions dans la détermination du développement des systèmes financiers a reçu une attention considérable dans

\footnotetext{
17 L'ensemble de ces variables est exprimé en logarithme.

18 Toutes les spécifications sont exemptes du risque d'auto-corrélation sérielle d'ordre 2 des termes d'erreur, ainsi que de celui de non validité des instruments internes utilisés.
} 
la littérature économique ${ }^{19}$. Les résultats conduisent à des conclusions nuancées. L'ouverture financière n'affecte pas de manière significative l'efficacité des banques locales et la taille des intermédiaires financiers bancaires et non bancaires. L'intégration financière internationale est, en revanche, à l'origine d'une baisse de l'activité des intermédiaires financiers. La déréglementation financière semble, enfin, impacter défavorablement le développement des marchés boursiers locaux, dans les économies émergentes et en développement.

\subsubsection{Taille des intermédiaires financiers}

Les résultats montrent que l'ouverture financière impacte d'une manière non statistiquement significative la taille (absolue et relative) des intermédiaires financiers bancaires et non bancaires. Ce résultat est vérifié quel que soit l'indicateur d'ouverture financière utilisé (de jure versus de facto) (tableau 5). Les résultats pour le ratio actifs banques de dépôts/banque centrale se trouvent légèrement améliorés après introduction de variables institutionnelles dans les régressions estimées et ce, vu que la significativité des coefficients pour cet indice est augmentée (tableau 6). Nos résultats se révèlent en partie comparables à ceux de Calderón et Kubota (2009) qui trouvent un impact non significativement différent de zéro d'une variation de l'indice de Chinn et Ito de libéralisation du compte financier sur le ratio des passifs liquides du système financier. Les passifs liquides et les actifs bancaires (rapportés au PIB) sont, par ailleurs, d'après les auteurs et conformément à nos résultats, non significativement corrélés à la somme (rapportée au PIB) des stocks bruts d'avoirs et d'engagements extérieurs.

\subsubsection{Activité des intermédiaires financiers}

L'intégration financière internationale réduit, d'après les résultats des estimations, l'activité des intermédiaires financiers. Cet effet concerne l'ensemble des indicateurs de l'activité financière utilisés (tableaux 5 et 6$)^{20}$. Nos résultats contredisent ceux de Chinn et Ito (2002), Law et

\footnotetext{
${ }^{19}$ La théorie d'Acemoglu et al. (2005) stipule que les différences de qualité institutionnelle constatées entre pays expliquent fortement les écarts inter-pays enregistrés en matière de développement. Selon ces auteurs, les institutions économiques sont des décisions sociales choisies pour leurs conséquences (développement économique, développement financier, protection des droits de propriété, amélioration de la gouvernance des entreprises, protection des créanciers...). Elles déterminent les incitations et les contraintes des agents économiques. Demetriades et Andrianova (2004) font valoir, de leur côté, que la force des institutions (efficacité de la réglementation financière, niveau de primauté du droit...) détermine la probabilité d'échec ou de succès des réformes financières. De point de vue empirique également, plusieurs travaux considèrent la variable « institutions » parmi les déterminants du développement financier (Knack et Keifer (1995), Chinn et Ito (2002, 2006), Law et Demetriades (2004), Baltagi et al. (2007, 2009), McDonald et Schumacher (2007), Yartey (2007), Andrianova et al. (2011), Law (2008, 2009), Calderón et Kubota (2009), Law et Habibullah (2009), Pham (2010)). Chinn et Ito (2002, 2006) et Calderón et Kubota (2009) trouvent que les systèmes financiers avec un développement institutionnel/légal élevé sont ceux qui bénéficient (en moyenne) le plus de la libéralisation financière externe. McDonald et Schumacher (2007) mettent en avant le rôle de la protection des droits des créanciers dans l'approfondissement du système financier. Yang (2011) teste l'effet d'une variation de la démocratie sur le niveau de développement du système financier. Il montre la présence d'une association positive entre la démocratie et le développement du système bancaire. Cette relation fait toutefois défaut en ce qui concerne le développement du marché boursier.

${ }^{20}$ Les résultats sont statistiquement significatifs aux seuils de $5 \%$ et $10 \%$. Il convient, cependant, de noter que la significativité des résultats est moins bonne lorsque l'on utilise les mesures de facto de l'ouverture financière.
} 
Demetriades (2004) et Calderón et Kubota (2009) qui démontrent la présence d'une relation positive entre l'intégration financière internationale, d'une part, et les mesures de l'activité du système financier, d'autre part.

\subsubsection{Efficacité des banques de dépôts}

Contrairement aux prévisions de la littérature théorique (Levine, 1996), les résultats démontrent la présence d'un lien non statistiquement significatif entre l'efficacité des banques locales et le degré d'intégration financière internationale. Une hausse du degré d'ouverture financière - dejure comme de facto - agit, d'après les estimations, d'une manière non significative sur le niveau des frais généraux et celui des marges bénéficiaires des banques locales. Les travaux existants aboutissent à des conclusions empiriques contraires (Levine (2003), Calderón et Kubota (2009)) ${ }^{21}$. Ils démontrent le déclin du ratio des frais généraux et celui des marges nettes d'intérêts des banques avec une plus grande intégration effective de l'économie nationale au sein des marchés financiers mondiaux. Lorsque nous prenons en compte le degré de développement institutionnel dans nos spécifications sur le développement financier (tableau 6), il apparait que la corrélation entre le ratio des frais généraux et celui des stocks bruts d'avoirs et d'engagements extérieurs devient significativement négative.

\subsubsection{Développement du marché boursier}

Les indicateurs d'intégration financière internationale montrent des coefficients essentiellement négatifs significatifs (aux seuils de $10 \%$ et $5 \%$ ) lorsque nous testons les implications d'une telle politique sur le développement des marchés boursiers dans les pays émergents et en développement ${ }^{22}$. Ces résultats contredisent les prévisions de la littérature défendant la présence d'une relation positive entre l'ouverture financière et le développement du marché boursier (Demirguç-Kunt et Levine (1996), Levine et Zervos (1998a), Kim et Singhal (2000), FuchsSchündeln et Funke (2003), de la Torre et al. (2007)). Ils sont en ligne, cependant, avec les résultats obtenus par Ben Naceur et al. (2008) pour un panel de 11 pays de la région MENA entre 1979 et 2005. Ces auteurs montrent que l'ouverture du marché boursier exerce un impact significativement défavorable à court terme sur le développement de ce dernier. Nos résultats suggèrent la présence d'effets de seuil dans le développement du marché boursier afin que celui-ci bénéficie de l'accroissement du degré d'ouverture financière.

\footnotetext{
${ }^{21}$ Les conséquences de l'ouverture financière sur l'efficacité du système bancaire sont rarement étudiées moyennant une approche macroéconomique. La littérature empirique privilégie plutôt des approches microéconomiques pour étudier une telle question.

${ }^{22}$ En raison d'un nombre insuffisant d'observations, l'indicateur Kaopen n'a pas été utilisé pour le modèle des marchés boursiers au niveau des tableaux 5 et 6 .
} 
En résumé, nos estimations empiriques conduisent à des conclusions nuancées quant à la nature du lien entre intégration financière internationale et développement du système financier dans les économies émergentes et en développement.

\subsection{Etude de sensibilité et de robustesse}

Nous apportons quelques modifications au modèle de base afin de tester la sensibilité de nos résultats ${ }^{23}$.

Les estimations sur panel dynamique de l'équation (2) ont été refaites en introduisant une mesure alternative de l'instabilité macroéconomique. Nous gardons, à ce titre, la même spécification empirique de départ, sauf que nous remplaçons la variable « taux d’inflation » par celle du «taux d'intérêt réel » (ir) (Fry (1997), Law et Demetriades (2004)). Les résultats confirment ceux décelés avec le taux d'inflation comme variable de contrôle. Les conséquences de l'ouverture financière sur l'activité des intermédiaires financiers et le développement du marché boursier se montrent significativement négatives. Par ailleurs, conformément aux résultats préalablement dégagés, l'intégration financière internationale ne semble pas impacter significativement la taille et l'efficacité des intermédiaires financiers.

Des résultats similaires sont obtenus lorsque nous testons la sensibilité des conclusions à l'élimination des observations aberrantes du développement financier. Nous excluons les observations pour lesquelles l'écart (en valeur absolue) du taux de croissance de celui-ci par rapport à sa moyenne dépasse deux fois son écart type. L'objectif est de voir si les résultats précédents ne sont pas en réalité tirés ou influencés par de fortes variations dans les indicateurs de développement financier, variations pouvant être liées à des épisodes de boom du crédit, de bulles sur le marché boursier ou d'effondrement des prix des actifs financiers ${ }^{24}$. Les résultats

\footnotetext{
${ }^{23}$ L'ensemble des spécifications estimées lors de l'analyse de robustesse est exempt du risque d'auto-corrélation sérielle d'ordre 2 des termes d'erreur, ainsi que de celui de non validité des instruments internes utilisés. Afin d'alléger l'exposé des résultats et leur conférer plus de lisibilité, nous rapportons uniquement ceux des estimations relatives à : l'indice de Chinn et Ito de libéralisation du compte financier et la somme rapportée au PIB des stocks bruts d'avoirs et d'engagements extérieurs. Les résultats de l'analyse de robustesse ne sont pas reproduits mais ils sont disponibles sur le lien suivant : http://economix.fr/docs/1291/Supplements.pdf.

${ }^{24}$ L'élimination des valeurs aberrantes selon la procédure décrite ci-haut réduit le nombre d'observations d'un degré relativement faible (compris entre 0 et $12 \%$ ) lorsque le développement financier est mesuré par les indicateurs d'activité financière (dépôts bancaires (11 \% d'observations en moins), dépôts du système financier (12\% d'observations en moins), crédit privé (11\% d'observations en moins) et crédit bancaire (0\% d'observations en moins). L'exclusion des observations aberrantes affecte, au contraire, fortement la taille de l'échantillon quand il s'agit de mesurer le degré de développement financier par les indicateurs de taille de l'intermédiation financière $(88 \%$ d'observations en moins pour le ratio actifs banques de dépôts/banque centrale, $53 \%$ d'observations en moins pour le ratio des passifs liquides et $22 \%$ d'observations en moins pour celui des actifs bancaires). Ceci rend les résultats trouvés pour l'indicateur actifs banques de dépôts/banque centrale peu fiables et explique le fait que nous ne les rapportons pas au niveau de l'annexe. Enfin, l'élimination des valeurs aberrantes n'affecte nullement le nombre d'observations pour les indicateurs d'efficience du secteur bancaire ou de développement du marché boursier $(0 \%$ d'observations en moins). Ceci ne peut que témoigner de la relative stabilité de ces mesures - par rapport aux autres indicateurs de développement financier considérés - pour l'échantillon total de pays étudiés.
} 
attestent une nouvelle fois de la robustesse de ceux obtenus auparavant ${ }^{25}$. L'élimination des observations aberrantes du développement financier semble même améliorer d'une façon considérable la qualité des résultats. Plusieurs coefficients de la variable ouverture financière deviennent statistiquement significatifs alors qu'ils ne l'étaient pas lors des estimations précédentes.

De la même manière, nous avons voulu tester la robustesse de nos résultats à l'introduction d'une nouvelle variable de contrôle, à savoir celle de la «crise financière » (voir tableau 1 de l'annexe pour plus de détails sur cette variable muette). L'ouverture financière peut, en effet, avoir un impact négatif sur le développement financier, tout simplement parce qu'elle peut conduire à une instabilité financière qui peut déboucher sur une crise financière (Rancière et al. (2006 et 2008), Guillaumont et Kpodar (2006) et Eichengreen et al. (2011)). Ainsi, contrôler les crises financières lors de nos estimations peut probablement réduire (voire éliminer) l’impact négatif préalablement trouvé - de l'ouverture financière sur le développement financier. Cette hypothèse n'a pas été soutenue par nos nouvelles estimations. L'impact négatif auparavant décelé de l'ouverture financière sur l'activité des intermédiaires financiers et le développement du marché boursier est maintenu après contrôle des crises financières dans le modèle estimé. Plus encore, cet effet négatif se trouve renforcé et devient statistiquement significatif à des seuils intéressants, suite à l'introduction de la variable crise. Le contrôle de l'effet des crises financières conforte ainsi les résultats précédents. Il rend les résultats auparavant trouvés plus significatifs là où ils l'étaient déjà. Les résultats auparavant non significatifs de l'impact de l'ouverture financière sur l'efficacité des banques locales et la taille des intermédiaires financiers le restent toujours même après introduction de la crise.

Au final, l'effet négatif décelé de l'ouverture financière n'est pas dû à un effet associé des crises financières pouvant accompagner cette dernière.

Enfin, nous avons voulu voir si les résultats précédemment trouvés ne dissimulent pas en réalité des différences entre les pays de l'échantillon. Pour ce faire, nous avons procédé à la réestimation de l'équation (2) pour deux sous-groupes de pays de notre échantillon de départ, à savoir : les pays émergents et frontière, d'une part, et les pays les moins développés, d'autre part (voir listes $\mathrm{B}$ et $\mathrm{C}$ de l'annexe 2). Les résultats montrent clairement la présence de divergences significatives, entre les deux groupes de pays, eu égard aux effets de l'ouverture financière sur les indicateurs de développement financier. Conformément aux résultats de la littérature, il apparait

\footnotetext{
${ }^{25}$ L'analyse de robustesse menée ici concerne uniquement la relation entre le développement financier et l'ouverture financière de facto (stocks bruts d'avoirs extérieurs, stocks bruts d'engagements extérieurs et stocks bruts d'avoirs et d'engagements extérieurs). L'élimination des observations aberrantes du développement financier fait que les estimations du lien empirique entre celui-ci (le développement financier) et l'ouverture financière de jure (indice Kaopen de Chinn et Ito) ne sont plus possibles et ce, faute de nombre suffisant d'observations.
} 
clairement qu'une plus grande intégration financière internationale des pays émergents et frontière favorise, d'une manière significative, le développement des intermédiaires financiers. Cet effet est essentiellement constaté pour l'indice de Chinn et Ito de libéralisation du compte financier. Il n'en demeure pas moins que l'ouverture financière de facto est toute aussi déterminante pour le développement de l'intermédiation financière, certes d'une manière un peu moins souvent significative. Les résultats mettent, toutefois, en avant un impact non significativement différent de zéro de l'ouverture financière (qu'elle soit de jure ou de facto) sur la plupart des indicateurs de développement du marché boursier. A contrario, les résultats pour les pays les moins développés montrent que l'ouverture financière n'exerce pas d'effets statistiquement significatifs sur la taille et/ou l'activité des intermédiaires financiers. Le développement des intermédiaires financiers dans ces économies est probablement tributaire de facteurs autres que le degré d'ouverture financière.

En conclusion, la thèse selon laquelle l'ouverture financière favorise le développement des systèmes financiers domestiques, dans les pays émergents et en développement, n'est pas validée par notre étude empirique. Nos résultats montrent que la nature du lien entre l'ouverture financière et le développement financier varie, pour ces pays, selon qu'il s'agit d'étudier les conséquences sur la taille, sur l'efficacité ou sur le volume d'activité du système financier (intermédiaires financiers et marché boursier). L’intégration financière internationale des pays émergents et en développement semble ne pas impacter d'une manière significative l'efficacité des banques locales et la taille des intermédiaires financiers bancaires et non bancaires. Elle serait, en revanche, à l'origine d'une baisse de l'activité des intermédiaires financiers. Les conséquences d'une telle politique sur le développement du marché boursier se montrent - également significativement préjudiciables. Les résultats pour le sous-groupe de pays émergents et frontière se révèlent plus concluants. Ils défendent sans ambigüité l'hypothèse selon laquelle leur plus grande insertion dans la sphère financière mondiale favorise le développement du secteur financier (de l'intermédiation financière en particulier).

Après avoir étudié la relation entre l'ouverture financière et le développement financier, testons dans ce qui suit celle s'initiant entre les trois variables ouverture financière, développement financier et croissance.

\section{Intégration financière internationale, développement financier et croissance de long} terme : les enseignements d'un modèle à équations simultanées

Les résultats précédents laissent penser que le degré de développement financier peut constituer un canal de transmission entre l'ouverture financière et la croissance de long terme dans les pays émergents et en développement. Nous étudions, dans ce qui suit, la relation tripartite ouverture 
financière - développement financier - croissance moyennant l'estimation d'un modèle à équations simultanées. Contrairement aux estimations séparées, l'estimation d'un tel modèle présente l'avantage de permettre la prise en compte dans l'analyse de l'ensemble des interactions possibles pouvant exister entre les variables étudiées. Elle est donc plus convenable pour évaluer correctement la nature du lien entre ces trois variables. L'estimation d'un modèle à équations simultanées permet, par ailleurs, de vérifier la robustesse des conclusions précédentes.

\subsection{Présentation du modèle}

Le modèle que nous estimons se compose de deux équations. Le développement financier est une variable explicative au niveau de la première équation (équation de croissance). Elle se transforme en une variable expliquée au niveau de la seconde équation (équation qui explique le degré de développement financier et au sein de laquelle l'ouverture financière est introduite en tant que variable explicative) et ce, comme suit :

$$
\left\{\begin{array}{l}
y_{i t}-y_{i t-1}=\alpha_{0}+\gamma y_{i t-1}+\alpha_{1} \boldsymbol{D} \boldsymbol{F}_{i t}+\alpha_{2} X_{i t}+\mu_{i}+\lambda_{t}+\varepsilon_{i t} \\
\boldsymbol{D} \boldsymbol{F}_{i t}=\beta_{0}+\tau D F_{i t-1}+\beta_{1} \boldsymbol{O F}_{i t-1}+\beta_{2} Z_{i t-1}+\mu_{i}+\lambda_{t}+\zeta_{i t}
\end{array}\right.
$$

$y$ est le PIB réel par habitant, $D F$ est le degré de développement financier, OF est une mesure du degré d'ouverture financière ${ }^{26}, X$ et $Z$ représentent deux jeux de variables de contrôle. $\mu_{i}$ représente l'effet spécifique au pays, $\lambda_{t}$ l'effet spécifique au temps et $\varepsilon$ et $\zeta$ sont des termes d'erreur. Les indices $i$ et $t$ désignent respectivement les pays $(i=1,2 \ldots \mathrm{N})$ et les périodes $(\mathrm{t}=1$, $2 . . \mathrm{T})$.

\subsection{Principaux résultats}

Les résultats des estimations du modèle à équations simultanées (modèle (3)) sont présentés dans le tableau 7. Le principal constat qui se dégage est leur similitude avec ceux précédemment trouvés lors des estimations équation par équation. En premier lieu, le degré de développement du système financier domestique (intermédiation financière et marché boursier) apparait comme un déterminant significatif du taux de croissance du PIB réel par habitant dans les pays émergents et en développement. Conformément aux prévisions de la littérature et aux résultats empiriques précédents, la variable développement financier présente dans tous les cas étudiés un impact positif significativement différent de zéro. En second lieu, le degré d’intégration financière internationale influence négativement et significativement le niveau d'activité des intermédiaires financiers et le développement du marché boursier dans les pays émergents et en développement. Les implications de cette politique sur l'efficience bancaire se montrent de leur côté non significatives. L'estimation par la méthode des équations simultanées rend, enfin, le coefficient de

\footnotetext{
${ }^{26}$ Nous nous limitons à l'emploi d'une seule mesure du degré d'intégration financière internationale, à savoir celle de la valeur rapportée au PIB des stocks bruts d'avoirs et d'engagements extérieurs (assetliab). Un tel choix se justifie par le souci de vouloir assurer un nombre suffisamment élevé d'observations.
} 
la variable ouverture financière - dans l'équation de la taille des intermédiaires financiers statistiquement significatif au seuil de $5 \%$, ce qui améliore les résultats antérieurs.

Nous pouvons ainsi conclure que le développement financier présente un canal de transmission indirect via lequel l'ouverture financière pourrait impacter le rythme de croissance dans les pays émergents et en développement. Les résultats des estimations montrent - à titre d'exemple qu'une hausse de $10 \%$ de la valeur rapportée au PIB des stocks bruts d'avoirs et d'engagements extérieurs augmenterait de $0,6 \%$ le ratio des passifs liquides qui, à son tour, relève de $0,08 \%(0,6$ $\% * 0,137)$ le taux de croissance du PIB réel par habitant. Une hausse de même ampleur du degré d'ouverture financière de facto est susceptible, en revanche, de baisser les dépôts bancaires, le crédit privé, la valeur des actions échangées en bourse, le taux de rotation des actifs et le nombre de firmes cotées en bourse par habitant de, respectivement, $1,43 \%, 1 \%, 7,46 \%, 4,3 \%$ et 4,5\% . De pareilles baisses dans les indicateurs de développement financier diminuent à leur tour le taux de croissance économique de, respectivement, $0,06 \%, 0,05 \%, 0,15 \%, 0,13 \%$ et $0,09 \%$.

Nous effectuons des estimations similaires pour le sous-groupe de 30 pays émergents et frontière. Les résultats (tableau 8) confirment largement et améliorent fortement ceux des estimations précédentes effectuées équation par équation. Ils montrent que l’intégration financière internationale favorise le développement du secteur financier domestique. Le développement financier exerce, à son tour, un impact positif significatif sur la croissance du PIB réel par habitant. Quel que soit l'indicateur de développement financier employé, les variables développement financier et ouverture financière entrent - toutes les deux - avec des coefficients significativement positifs. La plupart des coefficients affichés pour ces deux variables sont significatifs au seuil de $1 \%$, attestant ainsi de l'importance du lien tripartite intégration financière internationale - développement financier - croissance pour les marchés émergents et frontière. Il appartient donc aux autorités nationales de ces pays de veiller à une plus grande ouverture de leurs économies aux flux financiers transfrontaliers. Le but est de pouvoir espérer une croissance plus rapide et mieux soutenue (via le canal du développement financier).

La principale conclusion qui peut être dégagée de l'analyse à équations simultanées est que le développement financier constitue un canal de transmission indirect entre l'intégration financière internationale et la croissance dans les pays émergents et en développement. Les estimations montrent que le degré d'ouverture financière affecte significativement celui de développement du système financier qui, à son tour, impacte significativement le rythme de croissance dans ces pays. Nos résultats viennent fournir une explication plausible à l'absence de liens statistiquement significatifs entre l'intégration financière internationale et la croissance dans bien des travaux empiriques. Ces travaux testent la relation entre les deux variables moyennant l'estimation d'une 
régression de croissance qui contrôle le niveau de développement financier (parmi les variables explicatives du modèle estimé). Nous avons démontré ici que c’est à travers cette variable - ce canal indirect et collatéral - que l'ouverture financière pourrait impacter le rythme de croissance. Par conséquent, si le développement financier est pris en compte dans l'analyse du lien ouverture financière - croissance, il est tout à fait attendu que l'impact résiduel estimé de l'ouverture financière (sur la croissance) ne soit statistiquement significatif.

\section{Conclusion}

Dans cet article, nous avons cherché à voir dans quelle mesure le développement du système financier domestique constitue un déterminant clé de la croissance de long terme dans les pays émergents et en développement et, par conséquent, un canal de transmission indirect entre l'intégration financière internationale et la croissance économique dans ces pays. Les investigations menées concernent 112 pays émergents et en développement entre 1975 et 2007. Les principaux résultats obtenus sont les suivants. En premier lieu, nous avons confirmé les prédictions de la littérature quant à la présence d'un lien positif entre le développement financier et la croissance de long terme dans les pays émergents et en développement. En deuxième lieu, contrairement aux résultats d'une abondante littérature empirique défendant une relation positive entre l'ouverture financière et le développement financier, nous avons démontré qu'une telle relation est nuancée dans le cas des pays émergents et en développement. En effet, la nature de cette relation dépend du choix de l'indicateur de développement financier. Nos estimations concluent toutefois clairement et dans leur plus grande majorité à des effets négatifs importants de l'ouverture financière sur l'activité des intermédiaires financiers et le développement des marchés boursiers pour ces économies. Nos résultats sont robustes à une batterie de tests, notamment à l'introduction de la variable crises financières. Il convient cependant de souligner que nos résultats se sont révélés très concluants pour les pays émergents et frontière. Pour ces pays, nous avons identifié une relation positive entre l'ouverture financière et le développement financier. En troisième lieu, l'estimation du modèle à équations simultanées a permis de conclure que le développement financier constitue bien un canal de transmission indirect par lequel l'intégration financière internationale pourrait impacter le rythme de croissance de long terme dans les pays émergents et en développement. Le degré d'ouverture financière de ces pays semble affecter d'une manière significative celui de développement du système financier domestique qui, à son tour, altèrerait significativement la croissance de long terme de ces pays.

Les résultats obtenus dans cette étude peuvent être prolongées dans trois directions principales. En premier lieu, en prenant appui sur la littérature en termes d'effets de seuil (Kose et al. (2011), Allegret et Azzabi (2012 et 2013)), il convient d'estimer dans quelle mesure certaines 
caractéristiques structurelles - telles que le niveau de développement financier, la qualité des institutions locales et le degré d'ouverture commerciale - conditionnent la réponse du développement financier à une plus forte intégration financière internationale. En deuxième lieu, à partir des travaux ayant analysé les liens entre libéralisation financière et crise (Kaminsky et Schmukler (2008)), il est possible d'étudier l'influence de la composante instabilité financière sur le lien tripartite ouverture financière - développement du système financier - croissance. Une dernière extension est relative à l'étude du degré de stabilité dans le temps de la connexion ouverture financière - développement financier. Dans cette perspective, on peut voir si le signe ou l'ampleur de cette relation varie entre une période de stabilité financière et une période de turbulence.

\section{Bibliographie}

ACEMOGLU D. et ZILIBOTTI F. (1997), "Was prometheus unbound by chance? Risk, diversification, and growth », Journal of Political Economy, vol. 105, n²4, p.709-751.

ACEMOGLU D., JOHNSON S. et ROBINSON J.A. (2005), «Institutions as the fundamental cause of long-run growth », in P. Aghion et S. Durlauf (éds.), Handbook of Economic Growth, vol. 1, North Holland-Elsevier, Amsterdam, p.386-472.

ALLEGRET J.P. et AZZABI S. (2012), " Développement financier, croissance de long terme et effets de seuil », Panoeconomicus, vol. 59, n` 5, p.553-581.

ALLEGRET J.P. et AZZABI S. (2013), « Financial development, threshold effects and convergence in developing and emerging countries », Economics Bulletin, vol. 33, n³, p. 1899-1921.

ALLEN F. (1993), "Stock markets and resource allocation», in C. Mayer et X. Vives (éds), Capital Markets and Financial Intermediation, Cambridge University Press, Cambridge, p.81-116.

ALLEN F. et GALE D. (2000), Comparing Financial Systems, MIT Press, Cambridge, Mass.

ANDRIANOVA S., DEMETRIADES P. et XU C. (2011), " Political economy origins of financial markets in Europe and Asia », World Development, vol. 39, n5, p.686-699.

ARELLANO M. et BOND S. (1991), "Some tests of specification for panel data: Monte Carlo evidence and an application to employment equations », Review of Economic Studies, vol. 58, n², p.277-297.

ARELLANO M. et BOVER O. (1995), « Another look at the instrumental-variable estimation of error-components models », Journal of Econometrics, vol. 68, $\mathrm{n}^{\circ} 1, \mathrm{p} .29-52$.

BALTAGI B., DEMETRIADES P. et LAW S.H. (2009), «Financial development and openness: evidence from panel data », Journal of Development Economics, vol. 89, n² 2, p.285-296.

BARRO R., MANKIW G. et SALA-I-MARTIN X. (1995), « Capital mobility in neoclassical models of growth », The American Economic Review, vol. 85, n¹, p.103-115.

BECK T. et DEMIRGÜÇ-KUNT A. (2010), « Financial institutions and market across countries and over times Data and analysis », The World Bank Economic Review, vol. 24, n¹, 77-92.

BECK T. et LEVINE R. (2004), «Stock markets, banks and growth: panel evidence », Journal of Banking and Finance, vol. $28, n^{\circ} 3$, p.423-442.

BECK T., LEVINE R. et LOAYZA N. (2000), « Finance and the sources of growth », Journal of Financial Economics, vol. $58, \mathrm{n}^{\circ} 1-2$, p.261-300.

BEN NACEUR S., GHAZOUANI S. et OMRAN M. (2008), « Does stock market liberalization spur financial and economic development in the MENA region? », Journal of Comparative Economics, vol. 36, n 4, p.673-693.

BENCIVENGA V. et SMITH B.D. (1991), "Financial intermediation and endogenous growth », Review of Economics Studies, vol. $58, \mathrm{n}^{\circ} 2$, p.195-209.

BENHABIB J. et SPIEGEL M.M. (2000), «The role of financial development in growth and investment », Journal of Economic Growth, vol. 5, nº 4, p.341-360.

BERGER A.N., DEYOUNG H. et UDELL G.F. (2000), «Globalization of financial institutions: evidence from cross-border banking performance », Brookings Wharton Papers on Financial Services, vol. 3, p.23-120.

BLUNDELL R. et BOND S. (1998), "Initial conditions and moment restrictions in dynamic panel data models », Journal of Econometrics, vol. 87, $\mathrm{n}^{\circ} 1$, p.115-143.

BUSSIEERE M. et FRATZSCHER M. (2008), «Financial openness and growth: short-run gain, long-run pain? », Review of International Economics, vol. 16, $\mathrm{n}^{\circ} 1$, p.69-95.

CALDERÓN C. et KUBOTA M. (2009), « Does financial openness lead to deeper domestic financial markets ? », World Bank Policy Research Working Paper, nº 4973. 
CHINN M.D. et ITO H. (2002), « Capital account liberalization, institutions and financial development: cross country evidence », NBER Working Paper, n8967.

CHINN M.D. et ITO H. (2006), «What matters for financial development? Capital controls, institutions, and interactions », Journal of Development Economics, vol. 81, n¹, p.163-192.

DE LA TORRE A., GOZZI J.C. et SCHMUKLER S.L. (2007), « Stock market development under globalization: whither the gains from reforms? ", Journal of Banking and Finance, vol. 31, nº, p.1731-1754.

DELL'ARICCIA G. et MARQUEZ R. (2004), "Information and bank credit allocation », Journal of Financial Economics, vol. 72, n¹, p.185-214.

DEMETRIADES P.O. et ANDRIANOVA S. (2004), "Finance and growth: what we know and what we need to know ", in C. Goodhart (éd.), Financial development and economic growth: explaining the links, Palgrave Macmillan, Basingstoke and New York, p.38-65.

DEMIRGÜÇ-KUNT A. et LEVINE R. (1996), « Stock market development and financial intermediation: stylized facts », World Bank Economic Review, vol. 10, n², p.291-322.

DJANKOV S., MCLIESH C. et SHLEIFER A. (2007), " Private credit in 129 countries », Journal of Financial Economics, vol. 84, n², p.299-329.

DOLLAR D. (1992), "Outward-oriented developing economies really do grow more rapidly: evidence from 95 LDCs, 1976-1985 », Economic Development and Cultural Change, vol. 40, n³, p.523-544.

EASTERLY W. et REBELO S. (1993), « Fiscal policy and economic growth: an empirical investigation », Journal of Monetary Economics, vol. 32, n³, p.417-457.

EICHENGREEN B., GULLAPALLI R. et PANIZZA U. (2011), "Capital account liberalization, financial development and industry growth: A synthetic view », Journal of International Money and Finance, vol. 30, n 6, p. 10901106.

FISCHER S. (1993), «The role of macroeconomic factors in growth », Journal of Monetary Economics, vol. 32, $\mathrm{n}^{\circ} 3$, p.485-511.

FRY M.J. (1997), «In defense of financial liberalization », The Economic Journal, vol. 107, n442, p.754-770.

FUCHS-SCHÜNDELN N. et FUNKE N. (2003), "Stock market liberalizations: financial and macroeconomic implications ", Review of World Economics, vol. 139, n4, p.730-761.

GOURINCHAS P.O. et JEANNE O. (2006), "The elusive gains from international financial integration », Review of Economic Studies, vol. 73, n³, p.715-741.

GREENWOOD J. et JOVANOVIC B. (1990), « Financial development, growth and the distribution of income », Journal of Political Economy, vol. 98, n5, p.1076-1107.

GUILLAUMONT S. et KPODAR R. (2006), " Développement financier, instabilité financière et croissance économique », Economie \& prévision, n 174, p. 87-111.

HANSEN L.P. (1982), "Large sample properties of generalized method of moment estimators », Econometrica, vol. $50, \mathrm{n}^{\circ} 4, \mathrm{p} .1029-1054$.

HUANG W. (2006), «Emerging markets financial openness and financial development », University of Bristol Discussion Paper, $\mathrm{n}^{\circ} 588$.

HUYBENS E. et SMITH B.D. (1999), «Inflation, financial markets and long-run real activity », Journal of Monetary Economics, vol. 43, n², p.283-315.

JAIN-CHANDRA S. (2002), "The impact of stock market liberalization on liquidity and efficiency in emerging stock markets ", Columbia University Working Paper.

KAMINSKY G.L. et SCHMUKLER S.L. (2008), « Short-run pain, long-run gain: financial liberalization and stock market cycles », Review of Finance, vol. 12, n², p.253-292.

KHAN M.S., SENHADJI A.S. et SMITH B. (2006), «Inflation and financial depth », Macroeconomic Dynamics, vol. 10, $\mathrm{n}^{\circ} 2, \mathrm{p} .165-182$.

KIM E.H. et SINGAL V. (2000), "Stock market openings: experience of emerging economies », Journal of Business, vol. $73, \mathrm{n}^{\circ} 1$, p. $25-66$.

KING R.G. et LEVINE R. (1993a), «Finance and growth: Schumpeter might be right », The Quarterly Journal of Economics, vol. 108, n³, p.717-738.

KING R.G. et LEVINE R. (1993b), «Finance, entrepreneurship and growth », Journal of Monetary Economics, vol. 32, $\mathrm{n}^{\circ} 3, \mathrm{p} .1-30$.

KLEIN M. et OLIVEI G. (2008), « Capital account liberalization, financial depth, and economic growth », Journal of International Money and Finance, vol. 27, n6, p.861-75.

KNACK S. et KEEFER P. (1995), «Institutions and economic performance: cross-country tests using alternative institutional measures ", Economics and Politics, vol. 7, n³, p.207-227.

KOSE M.A., PRASAD E.S. et TAYLOR A.D. (2011), "Thresholds in the process of international financial integration », Journal of International Money and Finance, vol. 30, n¹, p.147-179.

KOSE M.A., PRASAD E.S., ROGOFF K. et WEI S.J. (2009), « Financial globalization: a reappraisal », IMF Staff Papers, vol.56, $\mathrm{n}^{\circ} 1, \mathrm{p} .8-62$.

KPODAR K. (2007), Manuel d'initiation à Stata (Version 8), Centre d'Etudes et de Recherches sur le Développement International, Clermont-Ferrand. 
LA PORTA R., LOPEZ-DE-SILANES F., SHLEIFER A. et VISHNY R.W. (1997), « Legal determinants of external finance », Journal of Finance, vol. 52, n³, p.1131-1150.

LA PORTA R., LOPEZ-DE-SILANES F., SHLEIFER A. et VISHNY R.W. (1998), « Law and finance », Journal of Political Economy, vol. 106, nº, p.1113-1155.

LAEVEN L. et VALENCIA F. (2012), «Systemic banking crises database: an update », IMF Working Paper, n¹63.

LANE P.R. et MILESI-FERRETTI G.M. (2007), « The external wealth of nations Mark II: revised and extended estimates of foreign assets and liabilities, 1970-2004 », Journal of International Economics, vol. 73, n² 2, p.223-250.

LAW S.H. (2008), " Does a country's openness to trade and capital accounts lead to financial development? ", Asian Economic Journal, vol. 22, n², p.161-177.

LAW S.H. (2009), «Trade openness, capital flows and financial development in developing economies », International Economic Journal, vol. 23, n³, p.409-426.

LAW S.H. et DEMETRIADES P. (2004), « Capital inflows, trade openness and financial development in developing countries », MMF Research Group Conference Paper, n 38.

LAW S.H. et HABIBULLAH M.S. (2009), "The determinants of financial development: institutions, openness and financial liberalization », South African Journal of Economics, vol. 77, $\mathrm{n}^{\circ} 1, \mathrm{p} .45-58$.

LEVINE R. (1996), "Foreign banks, financial development, and economic growth », in C.E. Barfield (éd.), International Financial Markets: Harmonization versus Competition, AEI Press, Washington, p.224-254.

LEVINE R. (1999), «Law, finance, and economic growth », Journal of Financial Intermediation, vol. 8, n²1/2, p.36-67.

LEVINE R. (2003), "Denying foreign bank entry: implications for bank interest margins », Central Bank of Chile Working Paper, ${ }^{\circ} 222$.

LEVINE R. (2005), " Finance and growth: theory and evidence », in P. Aghion et S. Durlauf (éds.), Handbook of Economic Growth, vol. 1, North Holland-Elsevier, Amsterdam, p.865-934.

LEVINE R. et ZERVOS S. (1998a), "Capital control liberalization and stock market development », World Development, vol. 26, nº 7 , p.1169-1183.

LEVINE R. et ZERVOS S. (1998b), "Stock markets, banks, and economic growth », The American Economic Review, vol. $88, \mathrm{n}^{\circ} 3$, p.537-558.

LEVINE R., LOAYZA N. et BECK T. (2000), " Financial intermediation and growth: causality and causes », Journal of Monetary Economics, vol. 46, n¹, p.31-77.

MAURO P. (1995), «Corruption and growth », Quarterly Journal of Economics, vol. 110, n³, p.681-712.

MCDONALD C.A. et Schumacher L. (2007), "Financial deepening in sub-Saharan Africa: empirical evidence on the role of creditor rights protection and information sharing », IMF Working Paper, $\mathrm{n}^{\circ} 203$.

MISHKIN F.S. (2006), The Next Great Globalization: How Disadvantaged Nations can Harness their Financial Systems to Get Rich, Princeton University Press, Princeton.

MISHKIN F.S. (2009), « Why we shouldn’t turn our backs on financial globalization », IMF Staff Papers, vol. 56, n¹, p.139-170.

PEDRONI P. (1996), «Fully Modified OLS for heterogeneous cointegrated panels and the case of purchasing power parity ", Indiana University Working Paper, $\mathrm{n}^{\circ} 20$.

PEDRONI P. (2000), "Fully modified OLS for heterogeneous cointegrated panels », in B.H. Baltagi (eds.), Nonstationary panels, panel cointegration, and dynamic panels, Advances in Econometrics, vol. 15, Elsevier Science, p.93130.

PESARAN M.H. et SMITH R. (1995), «Estimating long-run relationships from dynamics heterogeneous panels », Journal of Econometrics, vol. 68, n¹, p.79-113.

PHAM T.H.H. (2010), «Financial development, financial openness and trade openness: new evidence », FIW Working Paper, nº0.

RAJAN R.G. et ZINGALES L. (2003), « The great reversals: the politics of financial development in the twentieth century », Journal of Financial Economics, vol. 69, n¹, p.5-50.

RANCIERE R., TORNELL A. et WESTERMANN F. (2006), « Decomposing the effects of financial liberalization: crises vs. growth », Journal of Banking and Finance, vol. 30, n¹2, p.3331-3348.

RANCIERE R., TORNELL A. et WESTERMANN F. (2008), "Systemic crises and growth », Quarterly Journal of Economics, vol. 123, n¹, p.359-406.

RAPPAPORT J. (2000), « How does openness to capital flows affect growth? », FRB of Kansas City Research Working Paper, $\mathrm{n}^{\circ} 00-11$.

REINHART CM. et ROGOFF KS. (2010), «From financial crash to debt crisis », NBER Working Paper, n 15795.

RODRIK D. (2003), In Search of Prosperity: Analytic Narratives on Economic Growth, Princeton University Press, Princeton.

ROODMAN D. (2006), « How to do xtabond2: an introduction to "difference" and "system" GMM in Stata », Center for Global Development Working Paper, n¹03.

ROODMAN D. (2009), "A note on the theme of too many instruments », Oxford Bulletin of Economics and Statistics, vol. $71, \mathrm{n}^{\circ} 1, \mathrm{p} .135-158$.

ROUSSEAU P.L. et WACHTEL P. (2000), «Equity markets and growth: cross-country evidence on timing and outcomes », 1980-1995, Journal of Business and Finance, vol. 24, n¹2, p.1933-1957. 
SACI K., HOLDEN K. et GIORGIONI G. (2009), "Does financial development affect growth? ", Applied Economics, vol. 41, n¹3, p.1701-1707.

SENGUPTA R. (2007), "Foreign entry and bank competition », Journal of Financial Economics, vol. 84, n² 2, p.502-528. SEVESTRE P. (2002), Econométrie des données de panel, Editions Dunod, Paris.

SHLEIFER A. et VISHNY R.W. (1993), "Corruption », Quarterly Journal of Economics, vol. 108, n³, p.599-617.

SVALERYD H. et VLACHOS J. (2002), « Markets for risk and openness to trade: how are they related », Journal of International Economics, vol. 57, $\mathrm{n}^{\circ} 2$, p.369-395.

YANG B. (2011), « Does democracy foster financial development? An empirical analysis », Economic Letters, vol. 112, $\mathrm{n}^{\circ} 3, \mathrm{p} .262-265$.

YARTEY C.A. (2007), «Well-developed financial intermediary sector promotes stock market development », Journal of Emerging Market Finance, vol. 6, n³, p.269-289. 


\section{Annexes \\ Annexe 1 : Présentation des variables}

Tableau 1 - Définition des variables

Définition

Variable « développement financier »

Passifs liquides

Actifs banques de dépôts/banque centrale

Actifs bancaires

Dépots bancaire

Dépôts du système financier

Crédits au secteur prive

Crédits bancaires

Marge nette d'intérêt

Frais généraux

Capitalisation,

Valeur échangée

Nombre de firmes cotée

Kaopen

Avoirs et engagements extérieurs

Avoirs exterieurs

PIB réel par tête

Education

Inflation

Taille du gouvernement

Prime du marché de change parallèle

Population
Variation des termes de l'échange

Variation des termes de l'échan

Indice des libertés civiles

Indice des libertés politique

Taux d'intérêt réel

Risque politique

Stabilité gouvernementale

Corruption

Règle de droit

Démocratie

Qualité de la bureaucratic

Droits des créanciers

Crises financières

\section{PIB par tête en dollars constants (2000).}

\section{Passifs liquides (\% PIB).}

Actifs des banques commerciales sur la somme de ces actifs plus ceux de la banque centrale.

Actifs bancaires (\% PIB).

Dépôts des banques commerciales (\% PIB).

Dépôts du système financier $(\% \mathrm{PIB})$.

Crédits au secteur privé (\% PIB).

Crédits bancaires au secteur privé (\% PIB).

Revenu net d'intérêts / actif total des banques.

Frais généraux des banques/actif total des banques.

Valeur totale des parts cotées en bourse (\% PIB).

Valeur totale des actions échangées en bourse (\% PIB)

Valeur totale des actions échangées en bourse rapportée à la valeur totale des actions cotées.

Nombre de firmes cotées en bourse par habitant.

\section{Variable « ouverture financière »}

Indice de Chinn et Ito de libéralisation du compte financier.

Stocks bruts d'avoirs et d'engagements
Stocks bruts d'avoirs extérieurs (\% PIB)

Stocks bruts d'engagements extérieurs (\% PIB).

Taux de scolistion pinate (\% brt).

la consommation (annuel \%).

Dences de gouvernement $(\%$ PIB).

政

ux de change sur le marché parallèle et le taux de change officiel en $\%$ de ce dernier.

Taux de croissance de la population.

Variation de l'indice des termes de l'échange de marchandises nets.

Cet indice contient trois composantes : la viabilité des contrats/le degré d'expropriation, le rapatriement des profits et les délais de paiement. Un score de $4(0)$ points correspond à un risque très faible (élevé).

Les critères de notation des pays se référent essentiellement au degré de liberté d'expression, de manifestation, d'éducation, de religion ...

Les critères de notation des pays se référent essentiellement au degré de transparence, de justesse et de liberté des élections.

Taux d'intérêt débiteur ajusté en fonction de l'inflation telle que mesurée par le déflateur du PIB.

Indicateur agréóé qui comprend : la stabilité gouvernementale, les conditions socio économiques, le profil des investissements, les conflits internes et externes,

h corruption, les militaires en politique, les tensions religieuses, la loi, les tensions ethniques, le respect de la démocratie et la qualité bureaucratique. Ies vales,

álevées (faibles) de cet indice indiquent un risque faible (élevé), la lo

Elle comprend trois composantes : l'unité gouvernementale, la force législative et le soutien populaire. Chacune de ces composantes obtient un score minimum (maximum) de 0 (4) points (risque très élevé (faible)).

Les notes sont attribuées suivant la corruption financière sur les licences d'exportation ou d'importation, les contrôles de change, taxes etc .... Des valeurs élevées (faibles) de cet indice indiquent une corruption faible (élevée).

Elle comprend deux composantes : (i) une évaluation de la puissance et de l'impartialité des systèmes judiciaires et légaux et (ii) une évaluation du respect de la loi. Un faible score indique une plus grande fragilité règlementaire.

C'est une mesure de la façon avec laquelle le gouvernement est réceptif à son peuple. Les points sont attribués sur la base du type de gouvernance que connait le pays en question. La mesure obtient un score minimum de 0 points (démocratie faible) et maximum de 6 points (démocratie élevée).

Les points sont attribués lorsque la bureaucratie est indépendante de la pression politique. Le score maximum est de 4 points.

Indice agrégé des droits des créanciers. L'indice varie de « 0 » (faible protection des droits des créanciers) à « 4 » (forte protection).

Variable muette qui prend la valeur de « 1 » s’il ya eu crise financière durant la sous période concernée et « 0 » sinon.
Sources

Becke et Demirgïc-Kunt (2010) Becke et Demirgüs-Kunt (2010) Beck, et Demirgïs-Kunt (2010) Beck, et Demirgus-Kunt (2010) Beck et Demirgiug-Kunt (2010) Beck et Demirgüs-Kunt (2010) Beck, et Demirgüs-Kunt (2010) Beck et Demirgusc-Kunt (2010) Becke et Demirging-Kunt (2010) Becke et Demirgiug-Kunt (2010) Becke et Demirgurs-Kunt (2010) Becke et Demingus-Kunt (2010) Beck et Demirgüs-Kunt (2010)

bttp://web.pdx.edu/ ito/Chinn-Ito_website.btm. Lane et Milesi-Ferretti (2007) Lane et Milesi-Ferretti (2007)

World Development Indicators (2008) orld Development Indicators (2008) Wrild Development Indians (2008) World Development Indicators (2008) Global Development Network Database (2001) World Development Indicators (2008) Wristion

International Country Risk Guide (ICRG) International Country Risk Guide (ICRG) World Development Indicators (2008)

International Country Risk Guide (ICR

International Country Risk Guide (ICRG)

International Country Risk Guide (ICRG)

International Country Risk Guide (ICRG)

International Country Risk Guide (ICRG)

International Country Risk Guide (ICRG) Djankov et al. (2007) Reinhart et Rogoff (2010) et Laeven et Valencia (2012) 


\section{Annexe 2 : Classement des pays de l'échantillon}

Liste A : Liste des pays de l'échantillon (112 pays émergents et en développement)

Albanie, Algérie, Angola, Argentine, Arménie, Bangladesh, Belize, Bénin, Bhoutan, Bolivie, Botswana, Brésil, Bulgarie, Burkina Faso, Burundi, Cambodge, Cameroun, Cap-Vert, République centrafricaine, Tchad, Chili, Colombie, République démocratique du Congo, République du Congo, Costa Rica, Côte d'Ivoire, Dominique, République dominicaine, Equateur, Egypte, Salvador, Éthiopie, Fidji, Gabon, Gambie, Géorgie, Ghana, Grenade, Guatemala, Guinée-Bissau, Guyane, Haïti, Honduras, Inde, Indonésie, République islamique d'Iran, Jamaïque, Jordanie, Kazakhstan, Kenya, Kirghizie, République démocratique populaire du Lao, Lettonie, Lesotho, Libye, Lituanie, ex-République yougoslave de Macédoine, Madagascar, Malawi, Malaisie, Maldives, Mali, Mauritanie, Île Maurice, Mexique, République de Moldova, Mongolie, Maroc, Mozambique, Népal, Nicaragua, Niger, Nigéria, Pakistan, Panama, Papouasie-Nouvelle-Guinée, Paraguay, Pérou, Philippines, Pologne, Roumanie, Rwanda, Samoa, Sénégal, Serbie, Seychelles, Sierra Leone, Îles Salomon, Afrique du Sud, Sri Lanka, Saint Kitts-et-Nevis, Sainte-Lucie, SaintVincent-et-les Grenadines, Soudan, Suriname, Royaume du Swaziland, République arabe syrienne, Tanzanie, Thailande, Timor-Leste, Togo, Tonga, Tunisie, Turquie, Ouganda, Uruguay, Vanuatu, Venezuela, Vietnam, République du Yémen, Zambie, Zimbabwe.

Liste B : Liste des pays émergents (MSCI Barra de juin 2009)

Afrique du Sud, Argentine, Bangladesh, Brésil, Bulgarie, Chili, Colombie, Egypte, Inde, Iles Maurice, Indonésie, Jordanie, Kenya, Kazakhstan, Lituanie, Malaisie, Maroc, Mexique, Nigeria, Pakistan, Pérou, Philippines, Pologne, Roumanie, Serbie, Sri Lanka, Thailande, Tunisie, Turquie, Vietnam.

Liste C : Liste des pays les moins développés (pays à « revenu bas» selon la classification de la Banque Mondiale de juillet 2008)

Bénin, Burkina Faso, Burundi, Cambodge, République centrafricaine, Tchad, République démocratique du Congo, Éthiopie, Gambie, Ghana, Guinée-Bissau, Haiti, République de Kirghize, République démocratique populaire du Lao, Madagascar, Malawi, Mali, Mauritanie, Mozambique, Népal, Niger, Rwanda, Sénégal, Sierra Leone, Tanzanie, Togo, Ouganda, République du Yémen, Zambie, Zimbabwe. 


\section{Annexe 3 : Résultats des estimations}

Tableau 2 - Développement des intermédiaires financiers et croissance économique ${ }^{\mathrm{a}}$ Echantillon : 112 pays émergents et en développement entre 1975 et 2007 (données quinquennales)

Méthodologie : Méthode des Moments Généralisés en système

\begin{tabular}{|c|c|c|c|c|c|c|c|c|c|c|}
\hline \multirow[b]{2}{*}{ Variables } & \multirow[b]{2}{*}{$\begin{array}{l}\text { Signe } \\
\text { attendu }\end{array}$} & \multicolumn{3}{|c|}{ TAILLE } & \multicolumn{4}{|c|}{ ACTIVITE } & \multicolumn{2}{|c|}{ EFFICIENCE } \\
\hline & & $\begin{array}{r}\text { Passifs } \\
\text { liquides } \\
\end{array}$ & $\begin{array}{r}\text { Actifs banques de } \\
\text { dépôts/banque centrale }\end{array}$ & Actifs bancaires & $\begin{array}{l}\text { Crédit } \\
\text { privé }\end{array}$ & $\begin{array}{r}\text { Crédit } \\
\text { bancaire } \\
\end{array}$ & $\begin{array}{r}\text { Dépôts } \\
\text { bancaires }\end{array}$ & $\begin{array}{r}\text { Dépôts du système } \\
\text { financier } \\
\end{array}$ & $\begin{array}{r}\text { Marge } \\
\text { d'intérêts } \\
\end{array}$ & $\begin{array}{r}\text { Frais } \\
\text { généraux } \\
\end{array}$ \\
\hline PIB initial & $(-)$ & $\begin{array}{r}-\mathbf{0 , 0 5 6 * * *} \\
(0,000)\end{array}$ & $\begin{array}{r}-\mathbf{0 , 0 6 8 * * *} \\
(0,000)\end{array}$ & $\begin{array}{r}-\mathbf{0 , 1 0 1 * * *} \\
(0,000)\end{array}$ & $\begin{array}{r}-\mathbf{0 , 1 0 6 * * *} \\
(0,000)\end{array}$ & $\begin{array}{r}-\mathbf{0 , 1 1 2} * * * \\
(0,000)\end{array}$ & $\begin{array}{r}-\mathbf{0 , 0 6 6 * * *} \\
(0,000)\end{array}$ & $\begin{array}{r}-\mathbf{0 , 0 6 4 * * *} \\
(0,000)\end{array}$ & $\begin{array}{r}-\mathbf{0 , 0 0 1} * * * \\
(0,000)\end{array}$ & $\begin{array}{r}\mathbf{0 , 0 2 8} \boldsymbol{*} * \boldsymbol{*} \\
(0,000)\end{array}$ \\
\hline DF & $(+)$ & $\begin{array}{r}\mathbf{0 , 1 7 9 * * *} \\
(6,84 \mathrm{e}-05)\end{array}$ & $\begin{array}{r}\mathbf{0 , 2 1 0} * * * \\
(0,009)\end{array}$ & $\begin{array}{r}\mathbf{0 , 1 3 3} * * * \\
(0,005)\end{array}$ & $\begin{array}{r}\mathbf{0 , 1 1 7 * * *} \\
(0,001)\end{array}$ & $\begin{array}{r}\mathbf{0 , 1 0 8 * * *} \\
(0,007)\end{array}$ & $\begin{array}{r}\mathbf{0 , 1 5 8 * * *} \\
(1,81 \mathrm{e}-05)\end{array}$ & $\begin{array}{r}\mathbf{0 , 1 6 4 * * *} \\
(6,75 \mathrm{e}-06)\end{array}$ & $\begin{array}{l}-\mathbf{0}, 311 * * * \\
(1,33 \mathrm{e}-06)\end{array}$ & $\begin{array}{r}-\mathbf{0 , 2 8 1 * * *} \\
(0,000)\end{array}$ \\
\hline prim & $(+)$ & $\begin{array}{r}0,088 \\
(0,419)\end{array}$ & $\begin{array}{r}\mathbf{0 , 2 0 1 * * *} \\
(0,007)\end{array}$ & $\begin{array}{l}\mathbf{0 , 1 8 2 *} \\
(0,089)\end{array}$ & $\begin{array}{l}\mathbf{0 , 1 9 5 *} \\
(0,068)\end{array}$ & $\begin{array}{l}\mathbf{0 , 2 1 1 *} \\
(0,065)\end{array}$ & $\begin{array}{r}0,119 \\
(0,380)\end{array}$ & $\begin{array}{r}0,113 \\
(0,420)\end{array}$ & $\begin{array}{r}0,119 \\
(0,182)\end{array}$ & $\begin{array}{r}0,172 \\
(0,111)\end{array}$ \\
\hline $\inf$ & $(-)$ & $\begin{array}{r}-0,002 \\
(0,889)\end{array}$ & $\begin{array}{r}-0,020 \\
(0,403)\end{array}$ & $\begin{array}{r}-0,008 \\
(0,678)\end{array}$ & $\begin{array}{r}-0,008 \\
(0,589)\end{array}$ & $\begin{array}{r}-0,010 \\
(0,551)\end{array}$ & $\begin{array}{r}0,005 \\
(0,647)\end{array}$ & $\begin{array}{r}0,007 \\
(0,531)\end{array}$ & $\begin{array}{l}-0,019 \\
(0,601)\end{array}$ & $\begin{array}{r}-0,050 \\
(0,171)\end{array}$ \\
\hline gov & $(-)$ & $\begin{array}{r}-\mathbf{0 , 1 4 2 *} \\
(0,092)\end{array}$ & $\begin{array}{r}-\mathbf{0 , 1 6 8 *} \\
(0,081)\end{array}$ & $\begin{array}{r}\mathbf{- 0 , 1 5 9 *} \\
(0,073)\end{array}$ & $\begin{array}{l}-0,161 \\
(0,112)\end{array}$ & $\begin{array}{r}-\mathbf{0 , 1 4 3 *} \\
(0,090)\end{array}$ & $\begin{array}{r}-\mathbf{0 , 2 1 6 * *} \\
(0,032)\end{array}$ & $\begin{array}{r}-\mathbf{0 , 2 0 5 *} \\
(0,051)\end{array}$ & $\begin{array}{l}-0,032 \\
(0,555)\end{array}$ & $\begin{array}{l}-0,003 \\
(0,972)\end{array}$ \\
\hline trade & $(+)$ & $\begin{array}{r}0,078 \\
(0,331)\end{array}$ & $\begin{array}{r}0,041 \\
(0,481)\end{array}$ & $\begin{array}{r}0,091 \\
(0,263)\end{array}$ & $\begin{array}{r}0,123 \\
(0,133)\end{array}$ & $\begin{array}{r}0,121 \\
(0,145)\end{array}$ & $\begin{array}{r}0,053 \\
(0,606)\end{array}$ & $\begin{array}{r}0,053 \\
(0,614)\end{array}$ & $\begin{array}{r}-0,0552 \\
(0,485)\end{array}$ & $\begin{array}{l}-0,202 \\
(0,132)\end{array}$ \\
\hline bmp & $(-)$ & $\begin{array}{r}-\mathbf{0 , 1 1 9 * * * *} \\
(0,000) \\
\end{array}$ & $\begin{array}{r}-\mathbf{0 , 0 9 2 * * *} \\
(0,001) \\
\end{array}$ & $\begin{array}{r}-\mathbf{0 , 1 1 8 * * *} \\
(0,000) \\
\end{array}$ & $\begin{array}{r}-\mathbf{0 , 0 9 6 * * *} \\
(0,007) \\
\end{array}$ & $\begin{array}{r}-\mathbf{0 , 0 8 9 * *} \\
(0,019) \\
\end{array}$ & $\begin{array}{r}-\mathbf{0 , 1 1 1 * * * *} \\
(0,001) \\
\end{array}$ & $\begin{array}{r}-\mathbf{0 , 1 1 2} * * * \\
(0,002) \\
\end{array}$ & - & (2) \\
\hline \multicolumn{2}{|l|}{$\overline{\text { Obse }}$} & 283 & 287 & 282 & 281 & 281 & 279 & 279 & 301 & 305 \\
\hline \multicolumn{2}{|c|}{ Nombre de groupes } & 99 & 100 & 99 & 99 & 99 & 99 & 99 & 91 & 92 \\
\hline \multicolumn{2}{|c|}{ Test de Hansen ( $p$-value $)$} & 0,177 & 0,375 & 0,346 & 0,468 & 0,403 & 0,104 & 0,110 & 0,952 & 0,792 \\
\hline \multicolumn{2}{|c|}{ Test AR (2) (p-value) } & 0,238 & 0,295 & 0,509 & 0,682 & 0,681 & 0,464 & 0,497 & 0,479 & 0,201 \\
\hline \multicolumn{2}{|c|}{ Nombre d'instruments } & 38 & 38 & 39 & 39 & 39 & 21 & 22 & 28 & 21 \\
\hline
\end{tabular}

Notes La variable dépendante est le taux de croissance du PIB réel par habitant. La période d'étude 1975 - 2007 est subdivisée en 7 sous-périodes de cinq années chacune. PIB initial = PIB réel par habitant de début de période, DF = indicateurs de développement financier (passifs liquides rapportés au PIB, actifs des banques commerciales sur la somme de ces actifs plus ceux de la banque centrale, actifs bancaires rapportés au PIB, crédits au secteur privé rapportés au PIB, crédits bancaires au secteur privé rapportés au PIB, dépôts bancaires, dépôts du système financier, marge d'intérêts, frais généraux), prim $=$ Taux de scolarisation primaire, inf $=$ Taux d'inflation, gov $=$ Dépenses gouvernementales de consommation finale rapportées au PIB, trade $=$ Taux d'ouverture commerciale et bmp = Prime du marché de change parallèle. Toutes les variables sont introduites en logarithme sauf l'inflation $(\log (1+$ taux d'inflation)). Toutes les régressions incluent des variables muettes temporelles et une constante. Pour le test de Hansen, l'hypothèse nulle indique que les instruments utilisés sont valides (non corrélés avec les résidus). Pour le test AR(2), l'hypothèse nulle indique l'absence de corrélation sérielle de second ordre des erreurs en différence. a $p$-value entre parenthèses; * significatif au seuil d'erreur de $10 \%$, ** significatif au seuil d'erreur de $5 \%$, *** significatif au seuil d'erreur de $1 \%$. 
Tableau 3 - Développement du marché boursier et croissance économique ${ }^{\mathrm{a}}$ Echantillon: 112 pays émergents et en développement entre 1975 et 2007 (données quinquennales) Méthodologie : Méthode des Moments Généralisés en système

\begin{tabular}{|c|c|c|c|c|}
\hline Variables & Signe attendu & Capitalisation & Valeur échangée & Rotation \\
\hline \multirow[t]{2}{*}{ PIB initial } & $(-)$ & $-0,055^{* * *}$ & $-0,064 * * *$ & $-0,020 * * *$ \\
\hline & & $(0,000)$ & $(0,000)$ & $(0,000)$ \\
\hline \multirow[t]{2}{*}{ DF } & $(+)$ & $0,052 * *$ & $0,042 * *$ & 0,044 \\
\hline & & $(0,033)$ & $(0,035)$ & $(0,654)$ \\
\hline \multirow[t]{2}{*}{ prim } & $(+)$ & 0,022 & $-0,014$ & $-0,026$ \\
\hline & & $(0,903)$ & $(0,967)$ & $(0,961)$ \\
\hline \multirow[t]{2}{*}{$\inf$} & $(-)$ & $-0,119 * *$ & $-0,153^{*}$ & $-0,170^{*}$ \\
\hline & & $(0,044)$ & $(0,061)$ & $(0,063)$ \\
\hline \multirow[t]{2}{*}{ gov } & $(-)$ & $-0,067$ & $-0,072$ & $-0,122$ \\
\hline & & $(0,606)$ & $(0,572)$ & $(0,578)$ \\
\hline \multirow[t]{2}{*}{ trade } & $(+)$ & 0,068 & 0,045 & 0,047 \\
\hline & & $(0,413)$ & $(0,469)$ & $(0,650)$ \\
\hline \multirow[t]{2}{*}{ bmp } & $(-)$ & $-0,040$ & $-0,018$ & $-0,055$ \\
\hline & & $(0,222)$ & $(0,583)$ & $(0,149)$ \\
\hline \multicolumn{2}{|c|}{ Observations } & 124 & 119 & 124 \\
\hline \multicolumn{2}{|c|}{ Nombre de groupes } & 52 & 52 & 52 \\
\hline \multicolumn{2}{|c|}{ Test de Hansen ( $p$-value) } & 0,157 & 0,195 & 0,121 \\
\hline \multicolumn{2}{|c|}{ Test AR (2) (p-value) } & 0,410 & 0,211 & 0,215 \\
\hline \multicolumn{2}{|c|}{ Nombre d'instruments } & 27 & 21 & 22 \\
\hline
\end{tabular}

\footnotetext{
Notes La variable dépendante est le taux de croissance du PIB réel par habitant. La période d'étude 1975 - 2007 est subdivisée en 7 sous-périodes de cinq années chacune. PIB initial = PIB réel par habitant de début de période, $\mathrm{DF}=$ indicateurs de développement financier (valeur totale des parts cotées rapportée au PIB, valeur totale des parts échangées rapportée au PIB, ratio de rotation), prim = Taux de scolarisation primaire, inf $=$ Taux d'inflation, gov $=$ Dépenses gouvernementales de consommation finale rapportées au PIB, trade $=$ Taux d'ouverture commerciale et bmp = Prime du marché de change parallèle. Toutes les variables sont introduites en logarithme sauf l'inflation (log $(1+$ taux d'inflation)). Toutes les régressions incluent des variables muettes temporelles et une constante. Pour le test de Hansen, l'hypothèse nulle indique que les instruments utilisés sont valides (non corrélés avec les résidus). Pour le test $\mathrm{AR}(2)$, l'hypothèse nulle indique l'absence de corrélation sérielle de second ordre des erreurs en différence. ${ }^{a} p$ value entre parenthèses ; $*$ significatif au seuil d'erreur de $10 \%$, ** significatif au seuil d'erreur de $5 \%$, *** significatif au seuil d'erreur de $1 \%$.
} 
Tableau 4 - Développement financier et croissance économique : contrôle de variables institutionnelles ${ }^{a}$ Echantillon : 112 pays émergents et en développement entre 1975 et 2007 (données quinquennales)

Méthodologie : Méthode des Moments Généralisés en système

\begin{tabular}{|c|c|c|c|c|c|c|c|c|c|c|c|c|c|c|c|c|}
\hline \multirow[b]{2}{*}{ Variables } & \multirow{2}{*}{$\begin{array}{c}\text { Signe } \\
\text { attendu }\end{array}$} & \multicolumn{5}{|c|}{ Passifs liquides } & \multicolumn{5}{|c|}{ Actifs banques de dépôts/banque centrale } & \multicolumn{5}{|c|}{ Actifs bancaires } \\
\hline & & Modèle 1 & Modèle 2 & Modèle 3 & Modèle 4 & Modèle 5 & Modèle 1 & Modèle 2 & Modèle 3 & Modèle 4 & Modèle 5 & Modèle 1 & Modèle 2 & Modèle 3 & Modèle 4 & Modèle 5 \\
\hline \multirow[t]{2}{*}{ PIB initial } & $(-)$ & $0,019 * * *$ & $0,031 * * *$ & $0,003 * * *$ & $-0,065 * * *$ & $-0,061 * * *$ & $0,013^{* * *}$ & $0,012 * * *$ & $0,009 * * *$ & $-0,055^{* * *}$ & $-0,059 * * *$ & $-0,005 * * *$ & $-0,001 * * *$ & $-0,028 * * *$ & $-0,097 * * *$ & $-0,094 * * *$ \\
\hline & & $(0,000)$ & $(0,000)$ & $(0,000)$ & $(0,000)$ & $(0,000)$ & (0), 000 & $(0,000)$ & $(0,000)$ & $(0,000)$ & $(0,000)$ & $(0,000)$ & $(0,000)$ & $(0,000)$ & $(0,000)$ & $(0,000)$ \\
\hline \multirow[t]{2}{*}{ DF } & $(+)$ & $0,119 *$ & $0,141 *$ & $0,114 *$ & $0,126 * *$ & $0,110 * *$ & $0,135 *$ & $0,131 *$ & $0,124 * *$ & $0,302 * *$ & $0,243^{*}$ & $0,086^{*}$ & $0,126 * *$ & $0,106 * *$ & $0,160 * * *$ & $0,156^{* * *}$ \\
\hline & & $(0,057)$ & $(0,089)$ & $(0,087)$ & $(0,014)$ & $(0,026)$ & $(0,075)$ & $(0,059)$ & $(0,013)$ & $(0,017)$ & $(0,056)$ & $(0,061)$ & $(0,015)$ & $(0,029)$ & $(0,002)$ & $(0,001)$ \\
\hline \multirow[t]{2}{*}{ prim } & $(+)$ & $-0,079$ & $-0,083$ & $-0,090$ & 0,149 & 0,135 & $-0,006$ & 0,007 & $-0,070$ & 0,141 & 0,123 & $-0,014$ & $-0,073$ & $-0,054$ & 0,153 & 0,141 \\
\hline & & $(0,537)$ & $(0,599)$ & $(0,349)$ & $(0,245)$ & $(0,319)$ & $(0,946)$ & $(0,942)$ & $(0,270)$ & $(0,411)$ & $(0,437)$ & $(0,911)$ & $(0,570)$ & $(0,422)$ & $(0,360)$ & $(0,322)$ \\
\hline \multirow[t]{2}{*}{$\inf$} & $(-)$ & $-0,064$ & $-0,047$ & $-0,051$ & $-0,003$ & $-0,009$ & $-0,0712 *$ & $-0,102 *$ & $-0,0328$ & $-0,006$ & $-0,013$ & $-0,071$ & $-0,058$ & $-0,044$ & 0,004 & 0,000 \\
\hline & & $(0,180)$ & $(0,323)$ & $(0,211)$ & $(0,769)$ & $(0,506)$ & $(0,058)$ & $(0,076)$ & $(0,242)$ & $(0,734)$ & $(0,497)$ & $(0,144)$ & $(0,180)$ & $(0,277)$ & $(0,782)$ & $(0,958)$ \\
\hline \multirow[t]{2}{*}{ gov } & $(-)$ & $-0,276 * * *$ & $-0,313 * * *$ & $-0,219 * *$ & $-0,223 * *$ & $-0,240 * * *$ & $-0,243 * * *$ & $-0,218^{* * *}$ & $-0,213 * *$ & $-0,223^{* *}$ & $-0,194 * *$ & $-0,291 * * *$ & $-0,310 * * *$ & $-0,197 * * *$ & $-0,210 * *$ & $-0,227 * * *$ \\
\hline & & $(0,000)$ & $(0,002)$ & $(0,022)$ & $(0,020)$ & $(0,009)$ & $(0,000)$ & $(0,003)$ & $(0,021)$ & $(0,032)$ & $(0,040)$ & $(6,32 \mathrm{e}-05)$ & $(0,000)$ & $(0,004)$ & $(0,020)$ & $(0,008)$ \\
\hline \multirow[t]{2}{*}{ trade } & $(+)$ & $-0,108$ & $-0,160$ & $-0,036$ & 0,133 & 0,147 & $-0,026$ & $-0,052$ & 0,034 & 0,030 & 0,087 & $-0,088$ & $-0,141$ & $-0,008$ & 0,054 & 0,061 \\
\hline & & $(0,214)$ & $(0,167)$ & $(0,657)$ & $(0,123)$ & $(0,114)$ & $(0,752)$ & $(0,624)$ & $(0,624)$ & $(0,664)$ & $(0,244)$ & $(0,219)$ & $(0,130)$ & $(0,922)$ & $(0,489)$ & $(0,465)$ \\
\hline \multirow[t]{2}{*}{ bmp } & $(-)$ & & & & $-0,0957 * * *$ & $-0,090 * * *$ & & & & $-0,102 * * *$ & $-0,091 * * *$ & & & & $-0,120 * * *$ & $-0,108 * * *$ \\
\hline & & & & & $(0,004)$ & $(0,007)$ & & & & $(0,000)$ & $(0,000)$ & & & & $(0,000)$ & $(0,001)$ \\
\hline \multirow[t]{2}{*}{ Institutions } & $(+)$ & 0,042 & 0,065 & $0,190^{*}$ & 0,000 & $-0,009$ & 0,029 & 0,081 & 0,172 & 0,110 & $-0,015$ & 0,034 & 0,023 & $0,255^{* *}$ & 0,053 & $-0,017$ \\
\hline & & $(0,225)$ & $(0,561)$ & $(0,093)$ & $(0,998)$ & $(0,844)$ & $(0,331)$ & $(0,139)$ & $(0,112)$ & $(0,244)$ & $(0,768)$ & $(0,356)$ & $(0,776)$ & $(0,013)$ & $(0,639)$ & $(0,667)$ \\
\hline \multicolumn{2}{|l|}{ Observations } & 315 & 339 & 339 & 280 & 280 & 316 & 338 & 341 & 281 & 281 & 314 & 338 & 338 & 279 & 279 \\
\hline \multicolumn{2}{|c|}{ Nombre de groupes } & 76 & 78 & 78 & 97 & 97 & 76 & 78 & 78 & 98 & 98 & 76 & 78 & 78 & 97 & 97 \\
\hline \multirow{2}{*}{\multicolumn{2}{|c|}{$\begin{array}{l}\text { Test de Hansen ( }(p \text {-value }) \\
\text { Test AR (2) (p-value })\end{array}$}} & 0,491 & 0,492 & 0,310 & 0,109 & 0,154 & 0,231 & 0,118 & 0,152 & 0,147 & 0,176 & 0,434 & 0,355 & 0,246 & 0,119 & 0,311 \\
\hline & & 0,738 & 0,839 & 0,489 & 0,370 & 0,280 & 0,110 & 0,239 & 0,138 & 0,484 & 0,317 & 0,463 & 0,476 & 0,488 & 0,849 & 0,745 \\
\hline \multicolumn{2}{|c|}{ Nombre d'instruments } & 27 & 24 & 31 & 24 & 24 & 38 & 38 & 31 & 24 & 24 & 24 & 24 & 27 & 24 & 24 \\
\hline
\end{tabular}

Notes La variable dépendante est le taux de croissance du PIB réel par habitant. La période d'étude $1975-2007$ est subdivisée en 7 sous-périodes de cinq années chacune. PIB initial = PIB réel par habitant de début de période, $\mathrm{DF}=$ indicateurs de développement financier (passifs liquides rapportés au PIB, actifs des banques commerciales sur la somme de ces actifs plus ceux de la banque centrale, actifs bancaires rapportés au PIB), prim = Taux de scolarisation primaire, inf = Taux d'inflation, gov = Dépenses gouvernementales de consommation finale rapportées au PIB, trade = Taux d'ouverture commerciale, bmp = Prime du marché de change parallèle, bq = Qualité de la bureaucratie (Modèle 1), cor = Corruption (Modèle 2), invt = Profil d'investissement (Modèle 3), libciv = Indices des libertés civiles (Modèle 4)et libpol = Indices des libertés politiques $($ Modèle 5). Toutes les variables sont introduites en logarithme sauf l'inflation (log (1+taux d'inflation)). Toutes les régressions incluent des variables muettes temporelles et une constante. Pour le test de Hansen, l'hypothèse nulle indique que les utilisés sont valides (non corrélés avec les résidus). Pour le test AR(2), l'hypothèse nulle indique l'absence de corrélation sérielle de second ordre des erreurs en différence. a p-value entre parenthèses; $*$ significatif au seuil d'erreur de $10 \%$, ** significatif au seuil d'erreur de $5 \%$,*** significatif au seuil d'erreur de $1 \%$. 
Tableau 4bis - Développement financier et croissance économique : contrôle de variables institutionnelles (suite) ${ }^{a}$ Echantillon : 112 pays émergents et en développement entre 1975 et 2007 (données quinquennales)

Méthodologie : Méthode des Moments Généralisés en système

\begin{tabular}{|c|c|c|c|c|c|c|c|c|c|c|c|}
\hline \multirow[b]{2}{*}{ Variables } & \multirow{2}{*}{$\begin{array}{c}\text { Signe } \\
\text { attendu } \\
\end{array}$} & \multicolumn{5}{|c|}{ Crédit privé } & \multicolumn{5}{|c|}{ Crédit bancaire } \\
\hline & & Modèle 1 & Modèle 2 & Modèle 3 & Modèle 4 & Modèle 5 & Modèle 1 & Modèle 2 & Modèle 3 & Modèle 4 & Modèle 5 \\
\hline \multirow[t]{2}{*}{ PIB initial } & $(-)$ & $-0,017 * * *$ & $-0,012 * * *$ & $-0,032 * * *$ & $-0,120 * * *$ & $-0,115^{* * *}$ & $-0,017 * * *$ & $-0,008 * * *$ & $-0,027 * * *$ & $-0,114 * * *$ & $-0,112 * * *$ \\
\hline & & $(0,000)$ & $(0,000)$ & $(0,000)$ & $(0,000)$ & $(0,000)$ & $(0,000)$ & $(0,000)$ & $(0,000)$ & $(0,000)$ & $(0,000)$ \\
\hline \multirow[t]{2}{*}{ DF } & $(+)$ & $0,085^{* *}$ & $0,111 * *$ & $0,088^{* *}$ & $0,129 * * *$ & $0,125 * * *$ & $0,086^{* *}$ & $0,112 * *$ & $0,090 * *$ & $0,134 * * *$ & $0,126 * * *$ \\
\hline & & $(0,025)$ & $(0,017)$ & $(0,027)$ & $(0,001)$ & $(0,001)$ & $(0,037)$ & $(0,0177)$ & $(0,024)$ & $(0,003)$ & $(0,003)$ \\
\hline \multirow[t]{2}{*}{ prim } & $\overline{(+)}$ & 0,035 & $-0,009$ & $-0,078$ & $0,262 *$ & 0,231 & 0,035 & $-0,032$ & $-0,093$ & 0,219 & 0,205 \\
\hline & & $(0,778)$ & $(0,942)$ & $(0,316)$ & $(0,092)$ & $(0,115)$ & $(0,777)$ & $(0,816)$ & $(0,216)$ & $(0,196)$ & $(0,186)$ \\
\hline \multirow[t]{2}{*}{$\inf$} & $(-)$ & $-0,066$ & $-0,057$ & $-0,046$ & 0,003 & 0,001 & $-0,069$ & $-0,060$ & $-0,048$ & 0,002 & 0,000 \\
\hline & & $(0,123)$ & $(0,180)$ & $(0,301)$ & $(0,815)$ & $(0,937)$ & $(0,115)$ & $(0,153)$ & $(0,276)$ & $(0,879)$ & $(0,957)$ \\
\hline \multirow[t]{2}{*}{ gov } & $(-)$ & $-0,299 * * *$ & $-0,306 * * *$ & $-0,189 * * *$ & $-0,195 * *$ & $-0,210 * *$ & $-0,300 * * *$ & $-0,298 * * *$ & $-0,194 * * *$ & $-0,180 * *$ & $-0,191 * *$ \\
\hline & & $(1,76 \mathrm{e}-05)$ & $(0,000)$ & $(0,004)$ & $(0,031)$ & $(0,026)$ & $(8,73 e-06)$ & $(0,000)$ & $(0,003)$ & $(0,036)$ & $(0,027)$ \\
\hline \multirow[t]{2}{*}{ trade } & $(+)$ & $-0,072$ & $-0,116$ & 0,014 & 0,117 & 0,112 & $-0,069$ & $-0,117$ & 0,007 & 0,103 & 0,096 \\
\hline & & $(0,333)$ & $(0,185)$ & $(0,853)$ & $(0,146)$ & $(0,188)$ & $(0,347)$ & $(0,177)$ & $(0,922)$ & $(0,208)$ & $(0,269)$ \\
\hline \multirow[t]{2}{*}{ bmp } & $(-)$ & & & & $-0,081 *$ & $-0,081$ & & & & $-0,064$ & $-0,066$ \\
\hline & & & & & $(0,089)$ & $(0,109)$ & & & & $(0,185)$ & $(0,193)$ \\
\hline \multirow[t]{2}{*}{ Institutions } & $(+)$ & 0,031 & 0,008 & $0,269 * *$ & 0,069 & 0,006 & 0,032 & 0,008 & $0,257 * *$ & 0,055 & $-0,003$ \\
\hline & & $(0,396)$ & $(0,908)$ & $(0,022)$ & $(0,544)$ & $(0,893)$ & $(0,386)$ & $(0,909)$ & $(0,022)$ & $(0,624)$ & $(0,943)$ \\
\hline \multicolumn{2}{|c|}{ Observations } & 313 & 337 & 337 & 278 & 278 & 313 & 337 & 337 & 278 & 278 \\
\hline \multicolumn{2}{|c|}{ Nombre de groupes } & 76 & 78 & 78 & 97 & 97 & 76 & 78 & 78 & 97 & 91 \\
\hline \multicolumn{2}{|c|}{ Test de Hansen ( $p$-value $)$} & 0,650 & 0,405 & 0,149 & 0,169 & 0,304 & 0,596 & 0,312 & 0,199 & 0,165 & 0,304 \\
\hline \multirow{2}{*}{\multicolumn{2}{|c|}{ Test AR (2) (p-value) }} & 0,377 & 0,657 & 0,198 & 0,759 & 0,844 & 0,427 & 0,728 & 0,208 & 0,705 & 0,799 \\
\hline & & 24 & 24 & 27 & 24 & 24 & 24 & 24 & 27 & 24 & 24 \\
\hline
\end{tabular}

Notes La variable dépendante est le taux de croissance du PIB réel par habitant. La période d'étude 1975 - 2007 est subdivisée en 7 sous-périodes de cinq années chacune. PIB initial = PIB réel par habitant de début de période, $\mathrm{DF}=$ indicateurs de développement financier (crédits au secteur privé rapportés au PIB, crédits bancaires au secteur privé rapportés au PIB), prim = Taux de scolarisation primaire, inf $=$ Taux d'inflation, gov $=$ Dépenses gouvernementales de consommation finale rapportées au PIB, trade $=$ Taux d'ouverture commerciale, bmp $=$ Prime du marché de change parallèle, bq $=$ Qualité de la bureaucratie (Modèle 1), cor $=$ Corruption (Modèle 2), invt $=$ Profil d'investissement (Modèle 3 ), libciv $=$ Indices des libertés civiles $($ Modèle 4$)$ et libpol $=$ Indices des libertés politiques (Modèle 5). Toutes les variables sont introduites en logarithme sauf l'inflation (log (1+taux d'inflation)). Toutes les régressions incluent des variables muettes temporelles et une constante. Pour le test de Hansen, l'hypothèse nulle indique que les instruments utilisés sont valides (non corrélés avec les résidus). Pour le test AR(2), l'hypothèse nulle indique l'absence de corrélation sérielle de second ordre des erreurs en différence. a $p$-value entre parenthèses ; * significatif au seuil d'erreur de $10 \%$, ** significatif au seuil d'erreur de $5 \%$, *** significatif au seuil d'erreur de $1 \%$. 
Tableau 5 - Intégration financière internationale et développement du système financier Echantillon: 112 pays émergents et en développement entre 1975 et 2007 (données quinquennales)

Méthodologie : Méthode des Moments Généralisés en système

\begin{tabular}{|c|c|c|c|c|}
\hline Variable dépendante & $\begin{array}{r}\text { Kaopen } \\
(1) \\
\end{array}$ & $\begin{array}{r}\text { Asset } \\
(2) \\
\end{array}$ & $\begin{array}{r}\text { Liab } \\
(3) \\
\end{array}$ & $\begin{array}{r}\text { Assetliab } \\
\text { (4) } \\
\end{array}$ \\
\hline \multicolumn{5}{|l|}{ Taille des intermédiaires financiers } \\
\hline \multirow[t]{2}{*}{ Passifs liquides } & 0,019 & 0,075 & 0,074 & 0,060 \\
\hline & $(0,840)$ & $(0,592)$ & $(0,366)$ & $(0,600)$ \\
\hline \multirow[t]{2}{*}{ Actifs banques de dépôts/banque centrale } & 0,008 & 0,011 & 0,004 & 0,012 \\
\hline & $(0,932)$ & $(0,943)$ & $(0,933)$ & $(0,965)$ \\
\hline \multirow[t]{2}{*}{ Actifs bancaires } & 0,023 & 0,191 & 0,134 & 0,097 \\
\hline & $(0,794)$ & $(0,670)$ & $(0,364)$ & $(0,592)$ \\
\hline \multicolumn{5}{|l|}{ Activité des intermédiaires financiers } \\
\hline \multirow[t]{2}{*}{ Dépôts bancaires } & $-0,147 *$ & $-0,569$ & $-0,009$ & $-0,031$ \\
\hline & $(0,085)$ & $(0,263)$ & $(0,918)$ & $(0,840)$ \\
\hline \multirow[t]{2}{*}{ Dépôts du système financier } & $-0,173 *$ & $-0,555$ & $-1,637$ & $-0,075$ \\
\hline & $(0,093)$ & $(0,247)$ & $(0,340)$ & $(0,628)$ \\
\hline \multirow[t]{2}{*}{ Crédit au secteur privé } & $-0,110 * *$ & $-0,868^{*}$ & $-0,768$ & $-0,344$ \\
\hline & $(0,027)$ & $(0,058)$ & $(0,752)$ & $(0,537)$ \\
\hline \multirow[t]{2}{*}{ Crédit bancaire } & $-0,124 * *$ & $-0,833 *$ & $-0,426$ & $-0,463$ \\
\hline & $(0,030)$ & $(0,096)$ & $(0,285)$ & $(0,259)$ \\
\hline \multicolumn{5}{|l|}{ Efficience des intermédiaires financiers } \\
\hline \multirow{2}{*}{ Marge nette d'intérêts } & $-0,062$ & $-0,004$ & $-0,015$ & $-0,023$ \\
\hline & $(0,700)$ & $(0,993)$ & $(0,918)$ & $(0,924)$ \\
\hline \multirow[t]{2}{*}{ Frais généraux } & $-0,021$ & $-0,061$ & $-0,011$ & $-0,063$ \\
\hline & $(0,870)$ & $(0,722)$ & $(0,938)$ & $(0,749)$ \\
\hline \multicolumn{5}{|l|}{ Développement du marché boursier } \\
\hline \multirow[t]{2}{*}{ Capitalisation boursière } & - & $0,888^{*}$ & 0,493 & 0,190 \\
\hline & & $(0,069)$ & $(0,273)$ & $(0,682)$ \\
\hline \multirow[t]{2}{*}{ Rotation } & - & $-1,344 * *$ & $-1,387 *$ & $-1,269 *$ \\
\hline & & $(0,037)$ & $(0,052)$ & $(0,056)$ \\
\hline \multirow[t]{2}{*}{ Valeur échangée } & - & $-1,172 *$ & $-1,114 * *$ & $-1,300 *$ \\
\hline & & $(0,076)$ & $(0,015)$ & $(0,080)$ \\
\hline \multirow[t]{2}{*}{$\mathrm{N}^{\circ}$ Firmes cotées } & - & $-0,456$ & $-0,664 *$ & $-0,680 *$ \\
\hline & & $(0,265)$ & $(0,090)$ & $(0,087)$ \\
\hline
\end{tabular}

Notes La variable dépendante est le développement financier. La période d'étude 1975 - 2007 est subdivisée en 7 souspériodes de cinq années chacune. Kaopen $=$ Indice de Chinn et Ito de libéralisation du compte financier, Asset $=$ Stocks bruts d'avoirs extérieurs (rapportés au PIB), Liab = Stocks bruts d'engagements extérieurs (rapportés au PIB), Assetliab = Stocks bruts d'avoirs et d'engagements extérieurs (rapportés au PIB). Les spécifications estimées incluent les variables de contrôle suivantes : le développement financier retardé, le PIB réel par habitant, le taux d'inflation et le taux d'ouverture commerciale. Toutes les variables sont introduites en logarithme. Toutes les régressions incluent des variables muettes temporelles et une constante. La technique d'estimation employée est celle des moments généralisés sur panel dynamique en système. Afin de tester la validité des instruments utilisés, nous avons eu recours au test de Hansen (1982) de sur-identification des restrictions sur les moments. Nous avons aussi procédé au test d'auto-corrélation des erreurs d'Arellano et Bond (1991). ${ }^{a} p$-value entre parenthèses ; ${ }^{*}$ significatif au seuil d'erreur de $10 \%$, ** significatif au seuil d'erreur de $5 \%$, *** significatif au seuil d'erreur de $1 \%$. 
Tableau 6 - Intégration financière internationale et développement financier : $\underline{\text { contrôle des }}$ variables institutionnelles $^{\mathrm{a}}$

Echantillon: 112 pays émergents et en développement entre 1975 et 2007 (données quinquennales)

Méthodologie : Méthode des Moments Généralisés en système

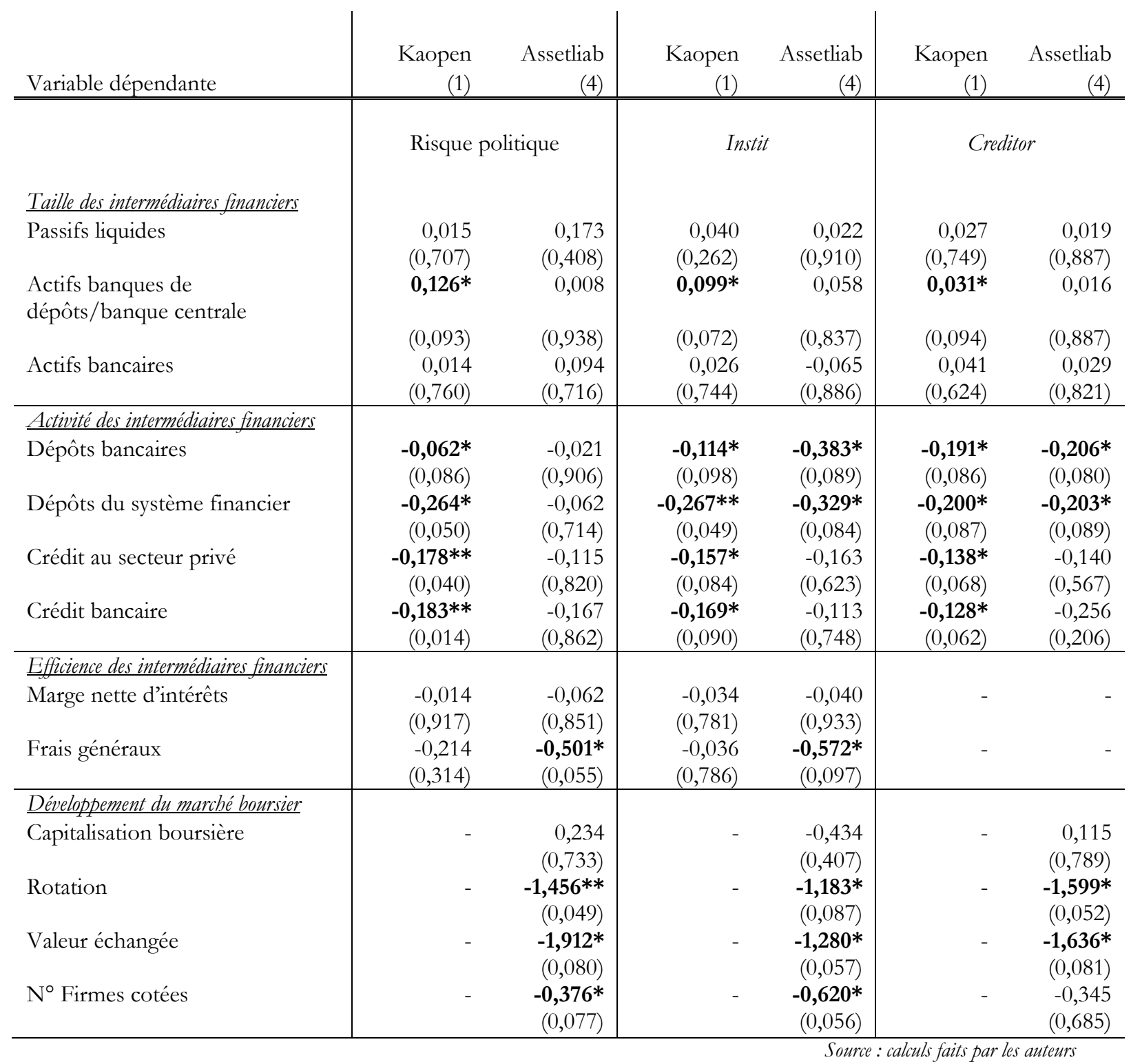

Notes La variable dépendante est le développement financier. La période d'étude 1975 - 2007 est subdivisée en 7 souspériodes de cinq années chacune. Kaopen = Indice de Chinn et Ito de libéralisation du compte financier, Assetliab = Stocks bruts d'avoirs et d'engagements extérieurs (rapportés au PIB). Les spécifications estimées incluent les variables de contrôle suivantes : le développement financier retardé, le PIB réel par habitant, le taux d'inflation, le taux d'ouverture commerciale et le taux de développement institutionnel (Risque politique $=$ indice de risque politique de l'ICRG, Creditor $=$ indice de protection des droits des créanciers, Instit = indice composite de «la corruption», «la démocratie », «la qualité de la bureaucratie», «le respect des lois» et «la stabilité du gouvernement»). Toutes les variables sont introduites en logarithme. Toutes les régressions incluent des variables muettes temporelles et une constante. La technique d'estimation employée est celle des moments généralisés sur panel dynamique en système. Afin de tester la validité des instruments utilisés, nous avons eu recours au test de Hansen (1982) de sur-identification des restrictions sur les moments. Nous avons aussi procédé au test d'autocorrélation des erreurs d'Arellano et Bond (1991). " $p$-value entre parenthèses ; * significatif au seuil d'erreur de $10 \%$, ** significatif au seuil d'erreur de $5 \%$, *** significatif au seuil d'erreur de $1 \%$. 
Tableau 7 - Ouverture financière, développement financier et croissance Echantillon : 112 pays émergents et en développement entre 1975 et 2007 (données quinquennales)

Méthodologie : Méthode des triples moindres carrés

\begin{tabular}{|c|c|c|c|c|c|c|c|c|c|}
\hline \multirow{2}{*}{ Variables } & \multirow[b]{2}{*}{$\begin{array}{c}\text { Signe } \\
\text { attendu }\end{array}$} & \multirow{2}{*}{$\begin{array}{r}\text { TAILLE } \\
\text { Passifs } \\
\text { liquides } \\
\end{array}$} & \multicolumn{2}{|c|}{ ACTIVITE } & \multirow{2}{*}{$\begin{array}{r}\text { EFFICIENCE } \\
\text { Marge nette } \\
\text { d'intérêts }\end{array}$} & \multicolumn{4}{|c|}{ MARCHE BOURSIER } \\
\hline & & & $\begin{array}{r}\text { Dépôts } \\
\text { bancaires }\end{array}$ & $\begin{array}{l}\text { Crédit } \\
\text { privé }\end{array}$ & & $\begin{array}{r}\text { Capitalisation } \\
\text { boursière }\end{array}$ & $\begin{array}{r}\text { Valeur } \\
\text { échangée }\end{array}$ & Rotation & $\begin{array}{r}\mathrm{N}^{\circ} \text { Firmes } \\
\text { cotées }\end{array}$ \\
\hline & & \multicolumn{8}{|c|}{ La variable dépendante est le taux de croissance du PIB réel par habitant } \\
\hline PIB initial & $(-)$ & $\begin{array}{r}-\mathbf{0 , 0 1 8} \boldsymbol{* * * *} \\
(0,000)\end{array}$ & $\begin{array}{r}\mathbf{0 , 0 1 0} \text { **** } \\
(0,000)\end{array}$ & $\begin{array}{r}-\mathbf{0 , 0 1 6 * * *} \\
(0,000)\end{array}$ & $\begin{array}{r}-\mathbf{0 , 0 0 1} * * * \\
(0,000)\end{array}$ & $\begin{array}{r}-\mathbf{0 , 0 0 7} * * * \\
(0,000)\end{array}$ & $\begin{array}{r}-\mathbf{0 , 0 0 3} * * * \\
(0,000)\end{array}$ & $\begin{array}{r}-\mathbf{0 , 0 0 9 * * *} \\
(0,000)\end{array}$ & $\begin{array}{r}-\mathbf{0 , 0 0 7 * * *} \\
(0,000)\end{array}$ \\
\hline DF & $(+)$ & $\begin{array}{r}\mathbf{0 , 1 3 7} * * \\
(0020)\end{array}$ & 0,042* & $\mathbf{0 , 0 5 2} * * *$ & $\begin{array}{r}-\mathbf{0 , 0 6 6 * * *} \\
(0,000)\end{array}$ & $\mathbf{0 , 0 3 0 * *}$ & 0,020* & $\mathbf{0 , 0 3 2} * *$ & $\mathbf{0 , 0 2 0}$ ** \\
\hline prim & $(+)$ & $\begin{array}{l}\mathbf{0 , 0 6 0 *} \\
(0,051)\end{array}$ & $\begin{array}{r}0,057 \\
(0,142)\end{array}$ & $\begin{array}{r}\mathbf{0 , 0 5 9} * * \\
(0,028)\end{array}$ & $\begin{array}{r}0,046 \\
(0,255)\end{array}$ & $\begin{array}{r}0,012 \\
(0,833)\end{array}$ & $\begin{array}{r}0,058 \\
(0,449)\end{array}$ & $\begin{array}{r}0,047 \\
(0,447)\end{array}$ & $\begin{array}{r}0,042 \\
(0,364)\end{array}$ \\
\hline $\inf$ & $(-)$ & $\begin{array}{r}0,011 \\
(0,383)\end{array}$ & $\begin{array}{r}-\mathbf{0 , 0 8 0} * * \\
(0,016)\end{array}$ & $\begin{array}{r}0,001 \\
(0,908)\end{array}$ & $\begin{array}{r}-\mathbf{0 , 0 5 5 * *} \\
(0,018)\end{array}$ & $\begin{array}{r}-\mathbf{0 , 0 6 8 * * *} \\
(0,003)\end{array}$ & $\begin{array}{r}-\mathbf{0 , 0 8 5 * * *} \\
(0,001)\end{array}$ & $\begin{array}{r}-\mathbf{0 , 0 7 1} * * * \\
(0,001)\end{array}$ & $\begin{array}{r}-\mathbf{0 , 0 8 6 * * *} \\
(3,51 \mathrm{e}-09)\end{array}$ \\
\hline gov & $(-)$ & $\begin{array}{r}-0,028 \\
(0,304)\end{array}$ & $\begin{array}{r}-\mathbf{0 , 0 5 6 *} \\
(0,054)\end{array}$ & $\begin{array}{l}-0,026 \\
(0,295)\end{array}$ & $\begin{array}{r}-\mathbf{0 , 0 5 2} * * \\
(0,010)\end{array}$ & $\begin{array}{r}-\mathbf{0 , 0 8 7} * \boldsymbol{*} * \\
(0,007)\end{array}$ & $\begin{array}{r}-\mathbf{0 , 1 0 0 * * *} \\
(0,009)\end{array}$ & $\begin{array}{r}-\mathbf{0 , 0 5 5} * \\
(0,078)\end{array}$ & $\begin{array}{r}-0,037 \\
(0,107)\end{array}$ \\
\hline trade & $(+)$ & $-0,025$ & $\begin{array}{r}-0,016 \\
(0459)\end{array}$ & 0,014 & $\begin{array}{r}0,009 \\
0,528)\end{array}$ & $\begin{array}{r}-0,004 \\
-0831)\end{array}$ & $\begin{array}{r}0,008 \\
(0711)\end{array}$ & 0,034 & $\begin{array}{l}-0,007 \\
\end{array}$ \\
\hline bmp & $(-)$ & $\begin{array}{r}(0,297) \\
-\mathbf{0 , 0 7 8} * * \\
(0,019) \\
\end{array}$ & $\begin{array}{r}(0,459) \\
-0,024 \\
(0,335) \\
\end{array}$ & $\begin{array}{r}(0,426) \\
-\mathbf{0 , 0 4 6 * *} \\
(0,018) \\
\end{array}$ & $(0,528)$ & $\begin{array}{r}(0,831) \\
-0,033 \\
(0,466) \\
\end{array}$ & $\begin{array}{r}(0,711) \\
0,002 \\
(0,963) \\
\end{array}$ & $\begin{array}{r}(0,108) \\
-0,033 \\
(0,476) \\
\end{array}$ & $(0,632)$ \\
\hline & & \multicolumn{8}{|c|}{ La variable dépendante est le développement financier } \\
\hline DF (ret) & $(+)$ & $\begin{array}{r}\mathbf{0 , 5 1 4} * * * \\
(0,000)\end{array}$ & $\begin{array}{r}\mathbf{0 , 7 6 9 * * *} \\
(0,000)\end{array}$ & $\begin{array}{r}\mathbf{0 , 7 5 7} * * * \\
(0,000)\end{array}$ & $\begin{array}{r}\mathbf{0 , 7 5 3} \boldsymbol{*} * \boldsymbol{*} \\
(0,000)\end{array}$ & $\begin{array}{r}\mathbf{0 , 7 4 9 * * *} \\
(0,000)\end{array}$ & $\begin{array}{r}\mathbf{0 , 7 0 3} * \boldsymbol{*} * \\
(0,000)\end{array}$ & $\begin{array}{r}\mathbf{0 , 6 7 2 * * * *} \\
(0,000)\end{array}$ & $\begin{array}{r}\mathbf{0 , 7 0 5 * * *} \\
(0,000)\end{array}$ \\
\hline $\begin{array}{l}\text { assetliab } \\
\text { (ret) }\end{array}$ & $(+/-)$ & $0,066 * *$ & $-0,143 * *$ & $-0,097^{*}$ & $-0,006$ & $-0,230$ & $-0,746^{*}$ & $-0,430^{*}$ & $-0,456 * * *$ \\
\hline & & $(0,029)$ & $(0,031)$ & $(0,081)$ & $(0,860)$ & $(0,176)$ & $(0,057)$ & $(0,070)$ & $(6,68 \mathrm{e}-05)$ \\
\hline ycap (ret) & $(+)$ & $\begin{array}{r}\mathbf{0 , 0 3 5 * *} \\
(0,038)\end{array}$ & $\begin{array}{r}0,055 \\
(0,117)\end{array}$ & $\begin{array}{r}\mathbf{0 , 0 8 1 * * *} \\
(0,002)\end{array}$ & $\begin{array}{l}-0,019 \\
(0,264)\end{array}$ & $\begin{array}{r}0,039 \\
(0,549)\end{array}$ & $\begin{array}{r}0,286 \\
(0,107)\end{array}$ & $\begin{array}{r}0,088 \\
(0,369)\end{array}$ & $\begin{array}{r}0,058 \\
(0,248)\end{array}$ \\
\hline $\inf (r e t)$ & $(-)$ & $\begin{array}{r}0,009 \\
(0,641)\end{array}$ & $\begin{array}{r}0,075 \\
(0,123)\end{array}$ & $\begin{array}{r}-0,012 \\
(0,763)\end{array}$ & $\begin{array}{r}\mathbf{0 , 2 7 9} * * * \\
(2,62 \mathrm{e}-07)\end{array}$ & $\begin{array}{r}-0,108 \\
(0,346)\end{array}$ & $\begin{array}{r}-0,225 \\
(0,304)\end{array}$ & $\begin{array}{r}-0,072 \\
(0,661)\end{array}$ & $\begin{array}{r}0,457 * * * \\
(6,51 \mathrm{e}-07)\end{array}$ \\
\hline trade (ret) & $(+)$ & $\begin{array}{r}0,024 \\
(0,521)\end{array}$ & $\begin{array}{r}0,126 \\
(0,122)\end{array}$ & $\begin{array}{l}\mathbf{0 , 0 9 7 *} \\
(0,094)\end{array}$ & $\begin{array}{r}-\mathbf{0 , 0 7 5 *} \\
(0,058)\end{array}$ & $\begin{array}{r}\mathbf{0 , 3 1 7 * *} \\
(0,035)\end{array}$ & $\begin{array}{r}0,096 \\
(0,809)\end{array}$ & $\begin{array}{l}-0,097 \\
(0,657)\end{array}$ & $\begin{array}{r}\mathbf{0 , 7 0 6} * * * \\
(2,29 \mathrm{e}-10)\end{array}$ \\
\hline
\end{tabular}

Notes La période d'étude 1975 - 2007 est subdivisée en 7 sous-périodes de cinq années chacune. PIB initial = PIB réel par habitant de début de période, $\mathrm{DF}=$ indicateurs de développement financier (passifs liquides rapportés au PIB, dépôts bancaires rapportés au PIB, crédits au secteur privé rapportés au PIB, ratio de la marge nette d'intérêts, valeur des actions cotées rapportée au PIB, valeur des actions échangées rapportée au PIB, valeur des actions cotées rapportée à celle des actions échangées et nombre des firmes cotées en bourse par habitant), prim = Taux de scolarisation primaire, inf $=$ Taux d'inflation, gov $=$ Dépenses gouvernementales de consommation finale rapportées au $\mathrm{PIB}$, trade = Taux d'ouverture commerciale, bmp = Prime du marché de change parallèle, assetliab = Stocks bruts d'avoirs et d'engagements extérieurs rapportés au PIB et ycap = PIB réel par habitant. Toutes les variables sont introduites en logarithme (sauf l'inflation (log $(1+$ taux d'inflation)) dans la première équation). Toutes les régressions incluent des variables muettes temporelles et une constante. La p-value est entre parenthèses pour les tests sur les coefficients. $*$ significatif au seuil d'erreur de $10 \%$, ** significatif au seuil d'erreur de $5 \%$, *** significatif au seuil d'erreur de $1 \%$. 
Tableau 8 - Ouverture financière, développement financier et croissance : $\underline{\text { cas des pays }}$ émergents et frontière

Echantillon : 30 pays émergents et frontière entre 1975 et 2007 (données quinquennales)

Méthodologie : Méthode des triples moindres carrés

\begin{tabular}{|c|c|c|c|c|c|c|c|}
\hline \multirow[b]{2}{*}{ Variables } & & \multicolumn{2}{|c|}{ TAILLE } & \multicolumn{4}{|c|}{ ACTIVITE } \\
\hline & $\begin{array}{l}\text { Signe } \\
\text { attendu }\end{array}$ & $\begin{array}{l}\text { Passifs } \\
\text { liquides }\end{array}$ & $\begin{array}{r}\text { Actifs } \\
\text { bancaires }\end{array}$ & $\begin{array}{r}\text { Dépôts } \\
\text { bancaires }\end{array}$ & $\begin{array}{r}\text { Dépôts du } \\
\text { système } \\
\text { financier }\end{array}$ & $\begin{array}{l}\text { Crédit } \\
\text { privé }\end{array}$ & $\begin{array}{l}\text { Crédit } \\
\text { bancaire }\end{array}$ \\
\hline & & \multicolumn{6}{|c|}{ La variable dépendante est le taux de croissance du PIB réel par habitant } \\
\hline \multirow[t]{2}{*}{ PIB initial } & $(-)$ & $0,000 * * *$ & $-0,010 * * *$ & $0,002 * * *$ & $0,004 * * *$ & $-0,010 * * *$ & $-0,010 * * *$ \\
\hline & & $(0,000)$ & $(0,000)$ & $(0,000)$ & $(0,000)$ & $(0,000)$ & $(0,000)$ \\
\hline \multirow[t]{2}{*}{ DF } & $(+)$ & $0,076 * *$ & $0,094 * * *$ & $0,096 * * *$ & $0,084 * *$ & $0,066 * *$ & $0,104 * * *$ \\
\hline & & $(0,024)$ & $(0,001)$ & $(0,005)$ & $(0,018)$ & $(0,036)$ & $(0,000)$ \\
\hline \multirow[t]{2}{*}{ prim } & $(+)$ & 0,006 & $-0,019$ & $-0,025$ & $-0,032$ & $-0,049$ & $-0,045$ \\
\hline & & $(0,919)$ & $(0,745)$ & $(0,669)$ & $(0,578)$ & $(0,430)$ & $(0,454)$ \\
\hline \multirow[t]{2}{*}{$\inf$} & $(-)$ & $-0,102 * * *$ & $-0,084 * *$ & $-0,091 * * *$ & $-0,096 * * *$ & $-0,085 * *$ & $-0,070 * *$ \\
\hline & & $(0,002)$ & $(0,011)$ & $(0,006)$ & $(0,004)$ & $(0,019)$ & $(0,046)$ \\
\hline \multirow[t]{2}{*}{ gov } & $(-)$ & $-0,175 * * *$ & $-0,148 * * *$ & $-0,163 * * *$ & $-0,166 * * *$ & $-0,161 * * *$ & $-0,151 * * *$ \\
\hline & & $(6,11 \mathrm{e}-06)$ & $(4,81 \mathrm{e}-05)$ & $(1,06 \mathrm{e}-05)$ & $(9,93 \mathrm{e}-06)$ & $(3,02 \mathrm{e}-05)$ & $(5,01 \mathrm{e}-05)$ \\
\hline \multirow[t]{2}{*}{ trade } & $(+)$ & $-0,008$ & $-0,012$ & $-0,013$ & $-0,008$ & $-5,30 \mathrm{e}-05$ & $-0,019$ \\
\hline & & $(0,724)$ & $(0,590)$ & $(0,585)$ & $(0,718)$ & $(0,998)$ & $(0,413)$ \\
\hline \multirow[t]{3}{*}{ bmp } & $(-)$ & $-0,328 *$ & $-0,269$ & $-0,165$ & $-0,202$ & $-0,360 * *$ & $-0,268$ \\
\hline & & $(0,055)$ & $(0,122)$ & $(0,385)$ & $(0,284)$ & $(0,042)$ & $(0,134)$ \\
\hline & & \multicolumn{6}{|c|}{ La variable dépendante est le développement financier } \\
\hline \multirow[t]{2}{*}{$\mathrm{DF}$ (ret) } & $(+)$ & $0,800 * * *$ & $0,745^{* * *}$ & $0,799 * * *$ & $0,791 * * *$ & $0,719 * * *$ & $0,689 * * *$ \\
\hline & & $(0,000)$ & $(0,000)$ & $(0,000)$ & $(0,000)$ & $(0,000)$ & $(0,000)$ \\
\hline \multirow{2}{*}{$\begin{array}{l}\text { assetliab } \\
\text { (ret) }\end{array}$} & $(+/-)$ & $0,231 * * *$ & $0,265 * *$ & $0,239 * * *$ & $0,237 * * *$ & $0,242^{*}$ & $0,250^{*}$ \\
\hline & & $(0,001)$ & $(0,018)$ & $(0,003)$ & $(0,003)$ & $(0,076)$ & $(0,063)$ \\
\hline \multirow[t]{2}{*}{ ycap (ret) } & $(+)$ & $-0,034$ & 0,016 & $-0,021$ & $-0,023$ & 0,043 & 0,025 \\
\hline & & $(0,213)$ & $(0,711)$ & $(0,505)$ & $(0,470)$ & $(0,401)$ & $(0,616)$ \\
\hline \multirow[t]{2}{*}{$\inf$ (ret) } & $(-)$ & $-0,089$ & $-0,184 *$ & $-0,116$ & $-0,121$ & $-0,274 * *$ & $-0,220 *$ \\
\hline & & $(0,172)$ & $(0,065)$ & $(0,124)$ & $(0,108)$ & $(0,024)$ & $(0,070)$ \\
\hline \multirow[t]{2}{*}{ trade (ret) } & $(+)$ & $-0,043$ & $-0,074$ & $-0,067$ & $-0,057$ & $-0,029$ & $-0,008$ \\
\hline & & $(0,453)$ & $(0,400)$ & $(0,321)$ & $(0,395)$ & $(0,779)$ & $(0,937)$ \\
\hline
\end{tabular}

Notes La période d'étude 1975 - 2007 est subdivisée en 7 sous-périodes de cinq années chacune. PIB initial = PIB réel par habitant de début de période, DF = indicateurs de développement financier (passifs liquides rapportés au PIB, actifs bancaires rapportés au PIB, dépôts bancaires rapportés au PIB, dépôts du système financier rapportés au PIB, crédits au secteur privé rapportés au PIB et crédits bancaires au secteur privé rapportés au PIB), prim = Taux de scolarisation primaire, inf = Taux d'inflation, gov = Dépenses gouvernementales de consommation finale rapportées au PIB, trade $=$ Taux d'ouverture commerciale, bmp $=$ Prime du marché de change parallèle, assetliab = Stocks bruts d'avoirs et d'engagements extérieurs rapportés au PIB et ycap = PIB réel par habitant. Toutes les variables sont introduites en logarithme (sauf l'inflation $(\log (1+$ taux d'inflation)) dans la première équation). Toutes les régressions incluent des variables muettes temporelles et une constante. La p-value est entre parenthèses pour les tests sur les coefficients. * significatif au seuil d'erreur de $10 \%$, ** significatif au seuil d'erreur de $5 \%$, *** significatif au seuil d'erreur de $1 \%$. 